\title{
A 3D Micromechanical Multi-Loop Magnetometer Driven Off-Resonance by an on-Chip Resonator
}

\author{
Giacomo Laghi, Cristiano Marra, Paolo Minotti, Alessandro Tocchio, and Giacomo Langfelder, Member, IEEE,
}

\begin{abstract}
The work presents the principle and the complete characterization of a single-chip unit formed by MEMS magnetometers to sense the 3D magnetic field vector, and a Tang resonator. The three sensors, nominally with the same resonance frequency, are operated $200 \mathrm{~Hz}$ off-resonance through an $\mathrm{AC}$ current whose reference frequency is provided by the resonator, embedded in an oscillating circuit. The sensors gain is increased by adopting a current recirculation strategy using metal strips directly deposited on the structural polysilicon. At a driving value of $100 \mu A_{r m s}$ flowing in series through the three devices, the magnetometers show a sub $185 \mathrm{nT} / \sqrt{\mathrm{Hz}}$ resolution with a selectable bandwidth up to $50 \mathrm{~Hz}$. Over a $\pm 5 \mathrm{mT}$ full-scale range, the sensitivity curves show linearity errors lower than $0.2 \%$, with high cross-axis rejection and immunity to external accelerations. Under temperature changes, stability of the 200$\mathrm{Hz}$ difference between the magnetometers and the resonator frequency is within $55 \mathrm{ppm} / \mathrm{K}$. Offset is trimmed down to the $\mu \mathrm{T}$ range, with an overall measured Allan stability of about 100 nT at 20 s observation time.
\end{abstract}

Keywords-MEMS sensors, magnetometers, acceleration rejection, vibrations, navigation.

\section{INTRODUCTION}

$\mathbf{M}$ ICROELECTROMECHANICAL system (MEMS) based magnetometers have been under research consideration for more than a decade, showing continuously improving performance in terms of system resolution, fullscale range (FSR), bandwidth, linearity, cross axis rejection and power consumption. From most of these standpoints, such devices (see e.g. the performance in [1]-[4]) are rapidly becoming competitive with commonly adopted technologies like Hall-effect, magnetic tunnel junction (MTJ) and anisotropic magneto-resistance (AMR) devices [5], [6]. This achievement is due to the conception and refinement of new operating principles, like parametric amplification [7], internal thermal-piezoresistive amplification [8], nonlinear sensitivity enhancement [9], off-resonance operation [1] or frequency modulation (FM) [2]. From a technological standpoint, it is advantageous to rely on MEMS magnetometers compatible with processes used for accelerometers and gyroscopes, so to form single-chip, single-process multi-parameter, multi-axis inertial measurement units (IMUs). So, which are the limiting

This research received funding from the European Community under the ENIAC Nanoelectronics Framework Programme under grant agreement $n$. Lab4MEMS (325622-2).

G. Laghi, C.R. Marra, P. Minotti and G. Langfelder are with Dipartimento di Elettronica, Informazione e Bioingegneria, Politecnico di Milano, 20133 Milano, Italy (tel: +39-02-2399-3744, email: giacomo.laghi@polimi.it). A. Tocchio is with the Analog, MEMS and Sensors division, ST Microelectronics, 20010, Cornaredo, Milano, Italy factors that still restrain the use of Lorentz-force devices in commercially available products? To follow up this question, this paper extends the work anticipated in [1] both by deepening results and discussion on all the aforementioned performance for all the 3 axes, and by characterizing other relevant parameters, not discussed in [1], inherent to MEMS and IMUs, which are scale-factor stability under temperature changes, offset issues, and sensitivity to accelerations.

As a matter of fact, the Lorentz force corresponding to a FSR magnetic field of a few $\mathrm{mT}$, on a few-hundred- $\mu \mathrm{m}$-long beam, carrying a few hundred $\mu A_{r m s}$ current, is more than two orders of magnitude lower than an inertial force corresponding to e.g. a $20 \bar{g}$ (gravity units) FSR acceleration on a typical MEMS accelerometer [10], [11]. It is also at least one order of magnitude lower than Coriolis forces on a MEMS gyroscope driven with a $5 \mu \mathrm{m}$ amplitude around $20 \mathrm{kHz}$, for typical FSR angular rates of 2000 dps [12], [13]. This small force value should give the reader an idea on how sources of sensitivity drift, offset [14], and effects of external accelerations [15] deserve a careful attention.

The paper thus first presents the single-chip MEMS system, formed by 3 multi-loop magnetometers (one per sensing axis) and a Tang resonator [16], [17]. The fabrication process constraints are analyzed to deepen aspects of magnetometers design with respect to [1]. The resonator is designed within the same die of the sensors to provide the reference frequency for the generation of the AC Lorentz current in off-resonance mode [1], [18], [19]. The aim is to make the 200-Hz mode-split difference stable against temperature changes. By theoretically discussing and experimentally measuring noise density (about $185 \mathrm{nT} / \sqrt{\mathrm{Hz}}$ at $100 \mu A_{r m s}$ of drive current consumption), bandwidth (selectable up to $50 \mathrm{~Hz}$ ), linearity (errors lower than $0.2 \%$ up to $5 \mathrm{mT} \mathrm{FSR}$ ) and cross-axis rejection (about 35 $\mathrm{dB})$, the paper demonstrates a resolution per unit bandwidth, normalized to Lorentz current consumption per single axis of $6.6 \mu \mathrm{T} \cdot \mu A_{r m s} / \sqrt{\mathrm{Hz}}$. This result outperforms previous works on MEMS magnetometers and several AMR, Hall-effect and MTJ commercial devices as well.

Then, possible harmfulness of external accelerations is introduced with a numerical example. It is shown how the presented design shifts acceleration-sensitive modes to higher frequencies than magnetic field sensing modes, providing good immunity to environmental vibrations. No significant sensitivity or offset change is experimentally obtained when operating under different $\mathrm{AC}$ and $\mathrm{DC}$ accelerations in the order of a few $\bar{g}$. Further, measurement of the shift of the magnetometers and resonator frequencies under temperature changes is shown to verify the stability of the scale factor against temperature 
changes, resulting in a variation lower than $0.5 \%$ over a 90 $\mathrm{K}$ temperature testing range.

Finally, different offsets origin, peculiar to this structures, and their effects are discussed to justify the obtained 70-150 nT stability at $100 \mu A_{r m s}$ driving current. In terms of absolute values, it is shown how the offset is trimmed down to about 2 $\mu \mathrm{T}$ via electromechanical action.

The paper is organized as follows: Section 2 describes the magnetic field sensors, the resonator to provide the operating frequency, and the electronics. Section 3 derives the theoretical performance in terms of sensitivity and noise, which are verified in Section 4. Section 5 focuses on behavior under accelerations and temperature changes, and on offset sources, with associated experimental measurements. In the concluding Section, a detailed benchmark to state-of-the-art and recent literature achievements is reported.

\section{SYSTEM OVERVIEW}

This section introduces the design of the 3-axis, amplitude modulated (AM) Lorentz force magnetometer. The system is operated through a single current, injected off-resonance in series through the three sensors. The transduction mechanism is based on capacitive readout.

\section{A. Process constraints and resistive model}

The whole MEMS module is designed and fabricated using a surface micromachined, $22-\mu \mathrm{m}$-thick, epitaxial polysilicon process from ST Microelectronics, featuring deep reactive ion etching (DRIE) for structural parts definition and hydrofluoric acid attack for device release. The process is currently used for mass production of accelerometers and gyroscopes. The reader can find more details in [20], [21].

In order to define the desired current trajectory, the sole different expedient adopted with respect to the aforementioned process is in the use of the step for deposition of metal paths. During this step, a 600-nm thick Al metal layer can be deposited on top of the epitaxial polysilicon, before DRIE is performed. Generally, the metal is used for electrical interconnections and it is not deposited on top of suspended parts (as nothing motivates this choice for accelerometers or gyroscopes). For what concerns magnetometers, the deposition of metals on top of suspended parts can help in defining the desired trajectory that the injected current must follow. For this reason, Al metal paths on top of polysilicon movable parts (springs or frames) were adopted. Note that it was not possible to add a buffer, insulating layer, between $\mathrm{Al}$ and polysilicon, due to various technological reasons (need for added process steps; temperature and residual stresses constraints given by the buffer layer...). As a consequence, there are in practice several possible trajectories for the current injected into the magnetometers inlet to reach the current outlet, namely: the ideal trajectory, flowing completely in the metal path; a parasitic trajectory, flowing completely through the shortest polysilicon path; or several combinations thereof. The effective current trajectory depends on the resistivity of the used materials, and on the geometry of the device. As the former is defined by the process (Al square resistance: 0.04

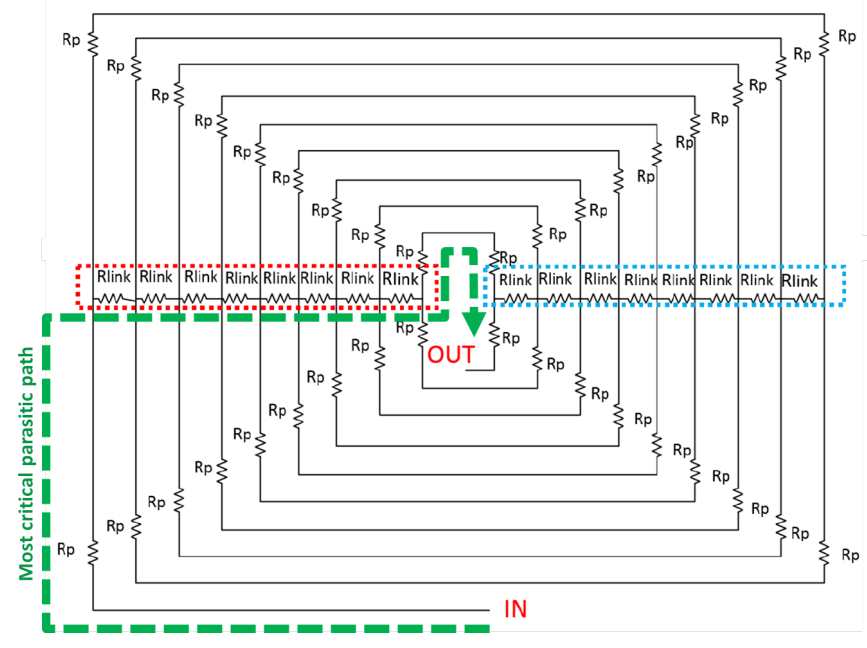

Fig. 1. Schematic representation of the resistive model of a generic multiloop magnetometer: the desired trajectory is a long spiral of low-resistivity Aluminum; possible leakage trajectories arise from short paths of highresistivity polysilicon (enclosed in dashed rectangles). The contact resistance between the layers was estimated to be $2.7 \mathrm{k} \Omega \cdot \mu \mathrm{m}^{2}$.

$\Omega_{/ \square}$; polysilicon square resistance: $20 \Omega_{/ \square}$ ), the geometry should be optimized to reject current flow through unwanted paths, as these leaks would not take part in the generation of the Lorentz force as desired. Note that even if the Al square resistance is much lower, the aspect ratio of its path, due to the adopted recirculation loops discussed below, can be orders of magnitude longer than for parasitic paths through polysilicon. This is shown in the general model given by Fig. 1: along the desired spiral path (from IN to OUT), there are contributions $\left(R_{p}\right)$ given by the $\mathrm{Al}$ resistance; along parasitic paths there are contributions given by the polysilicon resistance $\left(R_{\text {link }}\right)$; these links between adjacent spiral loops are unavoidable to keep them rigidly connected, so to obtain a single mechanical resonant sensing mode, as discussed in details below for the two kinds of devices presented in this work.

The following subsections give details (including strategies to minimize the current leakage) on magnetometers for in-plane (IP) field sensing (X- and Y-axis devices), on magnetometers for out-of-plane (OOP) magnetic field sensing (Z-axis), on the driving circuit (including the Tang resonator used to generate the AC frequency reference), and on the sensing electronics. All the MEMS devices lie in a single chip, packaged through glass-frit wafer-wafer bonding at a pressure of about 0.45 mbar. This pressure, slightly lower than the one adopted in [1], is used to lower the thermo-mechanical noise limit. In the following, the resonance frequencies of the $\mathrm{X}, \mathrm{Y}, \mathrm{Z}$ magnetometers and of the resonator will be named as $\left[f_{x}, f_{y}\right.$, $\left.f_{z}\right]=f_{m}$, and $f_{r}$ respectively.

\section{B. In-plane field sensing}

The device to sense an in-plane field along the Y-direction is shown in Fig. 2 (the device to sense the IP field along the Xdirection is a replica, rotated in plane by $90^{\circ}$ ). It is formed by 
four torsional beams, suspending a frame constituted by a 10loop spiral, where individual loops in the spiral are connected one another via thin links, to form the unique rigid frame. The structural frame is also anchored at the center, and nominally balanced along the rotation axis, so to minimize effects of accelerations along the sensing mode. On top of the frame, the Al metal is deposited so to define - though the thin links the effectively desired spiral trajectory of the Lorentz current. Suspending torsional beams have a width of $6.3 \mu \mathrm{m}$ and a length of $21.7 \mu \mathrm{m}$.

In operation, an $\mathrm{AC}$ current is made flow through the device from the anchor point labeled 'in' in Fig. 2a. The current follows the 27-mm-long spiral path (as indicated by $i$ and the arrows for the first two loops and for the final one) and exits from the anchor point labeled 'out'. In presence of a magnetic field $B_{y}$ along the Y-direction, a pair of Lorentz forces will act on average, as schematically indicated in the figure, determining a differential, out-of-plane, torsional motion. This is shown by the finite element method - FEM - simulation of Fig. 2b. Such a current recirculation directly generates a $9.5 \mathrm{x}$ improvement in sensitivity and resolution, as later discussed in Section III. According to SEM measurements, the width of the spiral polysilicon path is $6 \mu \mathrm{m}$ (as expected by design, accounting for nominal polysilicon overetch predictions), while the width of deposited Al strips is $3.3 \mu \mathrm{m}$ (about $40 \%$ lower than the value expected by design, probably due to an $\mathrm{Al}$ etching which was more aggressive than predicted).

Out-of-plane motion is detected via capacitance variation between the frame (which forms the top electrode) and two planar differential electrodes designed beneath the structure. The nominal vertical gap at rest between the rotor and the underneath planar electrodes is $1.8 \mu \mathrm{m}$. In the area corresponding to these bottom electrodes, the frame geometry is defined to increase the rest capacitance and the capacitance variation per unit displacement: Fig. $2 \mathrm{c}$ is a detail that shows how this is accomplished by adding rectangular blocks. The blocks are suitably holed not to impair the damping coefficient (and in turn thermomechanical noise) via extra squeezed-film damping.

Besides, maximization of the electrical resistance $R_{\text {link }}$ in between parallel, adjacent paths of the spiral (see again Fig. 1 ), is achieved by using serpentine links instead of direct links. This turns into a 4-fold larger resistance value along each link with respect to a direct connection, and thus minimizes leakage of Lorentz current between adjacent loops. Using SEM measured dimensions, the overall resistance of the 27-mm-long Al path is estimated to be $350 \Omega$, while the resistance along the shortest leaky path (as indicated in Fig. 1) is $5.5 \mathrm{k} \Omega$.

The device is designed to have the torsional mode (the first mode, to sense the Lorentz force as described above) at about $f_{y}=f_{m}=18.3 \mathrm{kHz}$, already including effects of electrostatic softening in operation. Like in gyroscopes, this value is at the margin of the typical acoustic disturbance bandwidth [22], which generally ends around $20 \mathrm{kHz}$. As detailed later in Section V, the mode sensitive to Z-axis accelerations/vibrations, as well as all other high-order modes, falls at frequencies larger than $32 \mathrm{kHz}$, thanks to the system
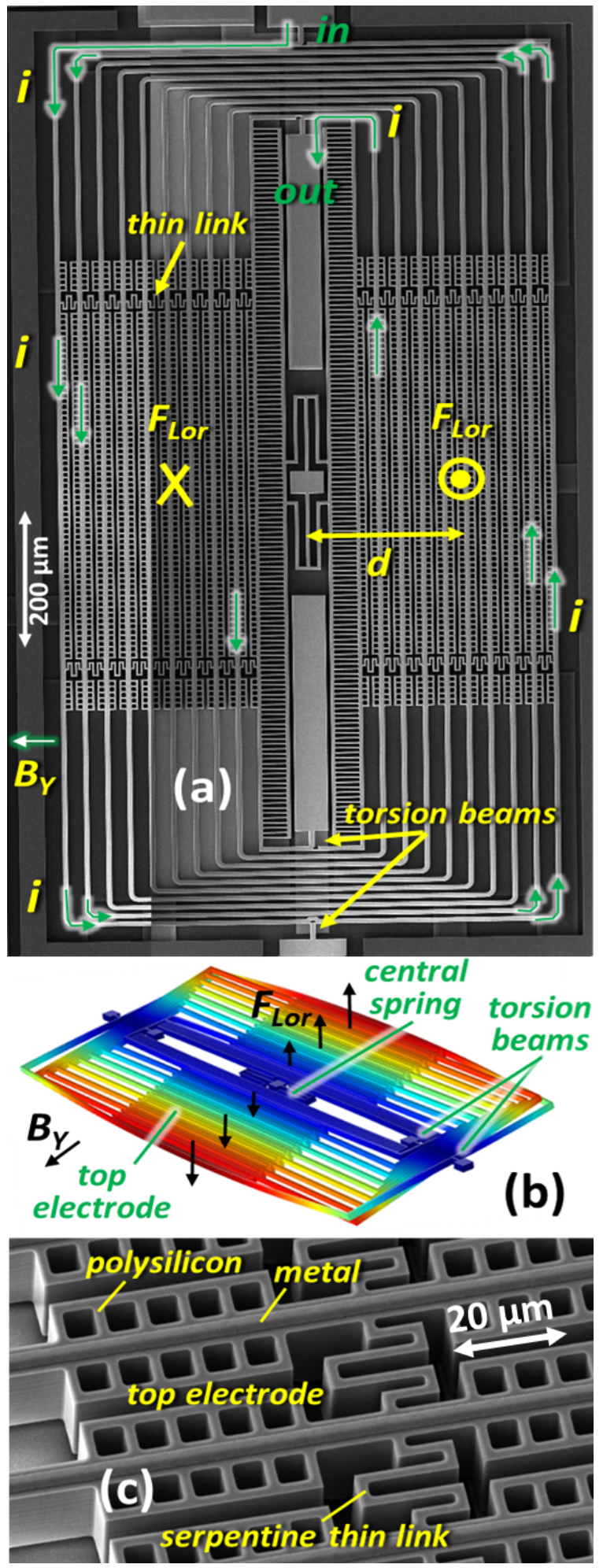

Fig. 2. Scanning electron microscope (SEM) images of the structure for IP field detection: (a) is a top view, showing the current inlet (in) and outlet (out) after the spiral recirculation; (b) is the first mode FEM corresponding to an OOP rotation; (c) is a detail showing the serpentine links connecting the spiral loops, and the top-electrode frame right above one bottom electrode. 
of springs designed and anchored at the device center. In this way, immunity to mechanical vibrations induced by acoustic speakers is maximized even if the sensing frequency $f_{m}$ is not strictly above the audio range. The overall device area, including the anchor points, is $1300 \mu \mathrm{m} \times 640 \mu \mathrm{m}$.

\section{Out-of-plane field sensing}

The device to sense a magnetic field along the out-of-plane, $\mathrm{Z}$-axis, direction is shown in Fig. 3. The device is derived from the one described in [23], differing in the optimized geometry of the readout electrodes, as later described. It is constituted by two symmetric halves, each formed by ten-beam springs, rigidly connected one another via straight thin links at their center. The springs suspend a battery of nested cells for inplane capacitive motion sensing. A diamond-shaped tuning fork, suspended at its ends by $410-\mu \mathrm{m}$-long clamped-clamped beams, connects the two halves. All along the 20 beams, a 40$\mathrm{mm}$-long $\mathrm{Al}$ path is deposited in order to create a spiral loop that makes the Lorentz current recirculate 10 times. Each beam has a width of $6 \mu \mathrm{m}$, again covered by a 3.3- $\mu \mathrm{m}$-wide Al strips. The overall Al loop resistance estimation is thus in the order of $480 \Omega$. According to the resistive model previously shown, the smaller-than-expected width for the metal paths generates in this case a non-negligible leakage of current through the (straight) links indicated in Fig. 3a. Their overall resistance along the most critical parasitic path turns out to be in the order of $1.5 \mathrm{k} \Omega$ : this lowers the improvement in sensitivity and resolution to a factor about $6.5 \mathrm{x}$ (instead of the nominal 10x determined by the number of recirculation loops). A serpentine link solution may be adopted also for this structure in future implementations.

In operation, an $\mathrm{AC}$ current is injected from the anchor point labeled 'in' in the figure. It follows the spiral path (as indicated by the arrows for the first two loops and for the final one) and exits from the anchor point labeled 'out'. In presence of a magnetic field $B_{z}$ along the OOP direction (the Z-axis), a pair of Lorentz forces excites the in-plane, anti-phase mode of the device. Its frequency, determined by the suspending springs and by the tuning fork geometry, is set at about $f_{z}=f_{m}=18.3$ $\mathrm{kHz}$ in operation (including the softening caused by electrodes biasing). The operation mode, shown by the FEM simulation of Fig. 3b, is thus set at a frequency nominally identical to those of X- and Y-axis devices. The anti-phase displacement is readout through a special geometry of sensing electrodes. Each fixed stator indeed features interspersed apertures that act as escape paths for squeezed gas, thus reducing, with respect to the twin device presented in [23], the damping coefficient and in turn the thermomechanical noise. According to electrical FEM simulations and to Montecarlo test particle damping simulations [24], this approach guarantees about the same capacitance variation per unit displacement and $45 \%$ lower damping with respect to a continuous stator [23]. The penalty of this new solution is the larger area occupied by each differential stator (the number of sensing cells fitting in the same overall area is roughly $85 \%$ lower). Details of these electrodes (holed stators) are visible in Fig. 3c: a 5.4 $\mu \mathrm{m}$ aperture is provided every $28.8 \mu \mathrm{m}$ of plate length. The
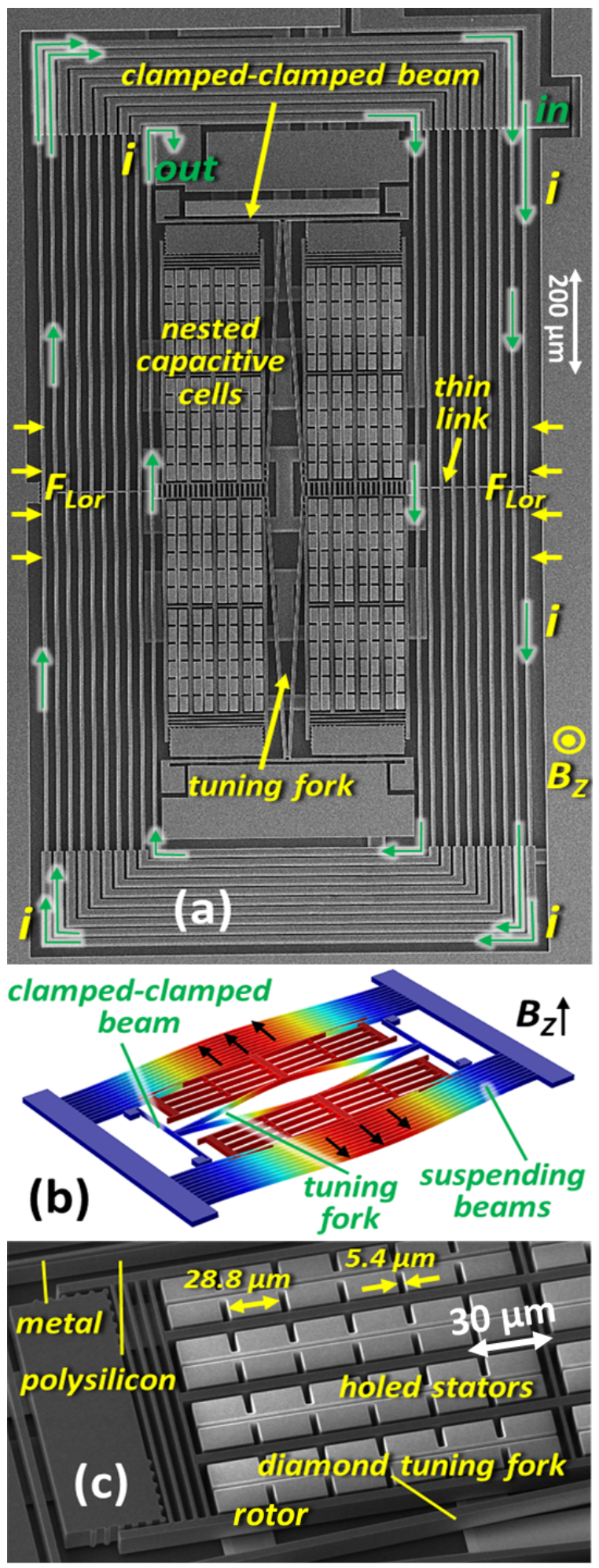

Fig. 3. SEM images of the structure for out-of-plane field detection: (a) is a top view, showing the current inlet and outlet after the spiral path recirculation; (b) is the first mode FEM showing the anti-phase mode excited by the Lorentz force; (c) is a detail showing the parallel-plate stators featuring interspersed escape paths for squeeze-film damping. 

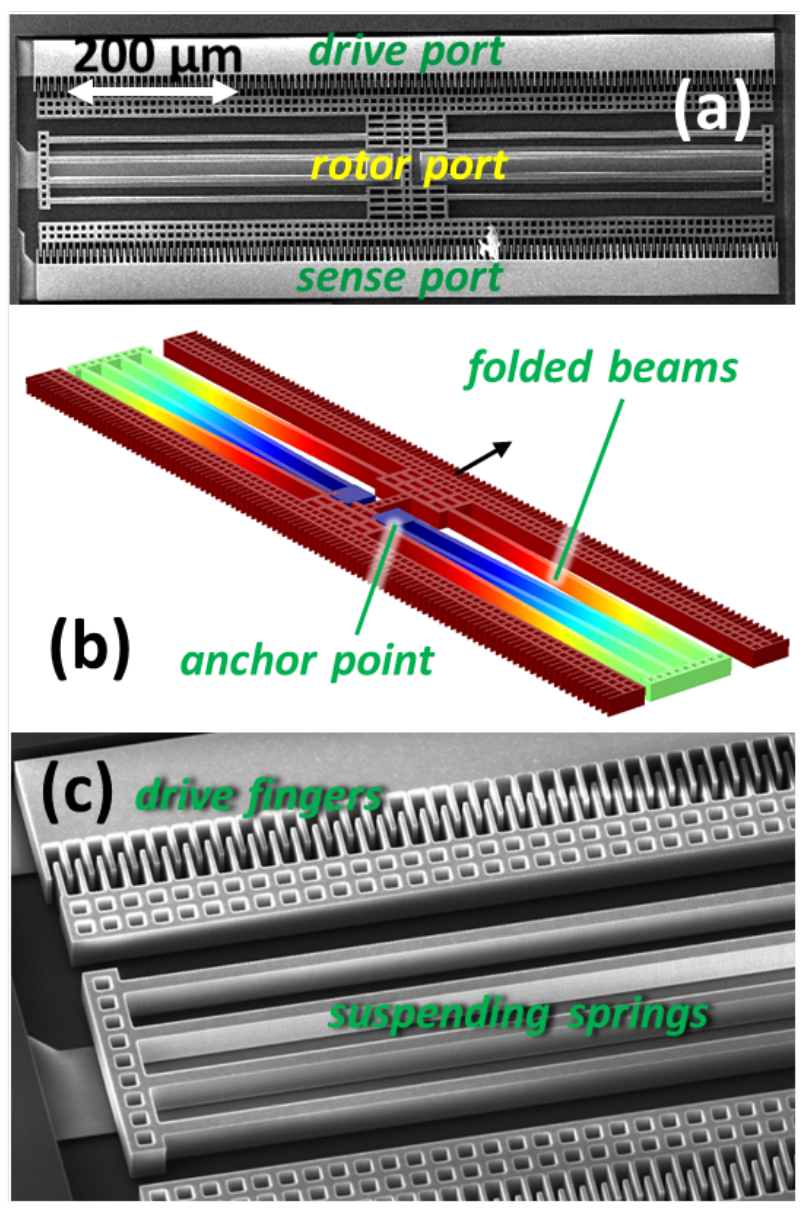

Fig. 4. SEM images of the Tang resonator used to provide the reference frequency in off-resonance operation: (a) is a top-view of the 3-port device (b) shows the first (in-plane translational) mode and (c) is a detail of springs and comb fingers.

former dimension is sized to be well larger than the gap, while the latter dimension is sized to be comparable to the structural thickness. For details on the dimensioning and advantages of this kind of parallel plates, the reader can refer to [25]. The nominal gap between the rotor and the stators is $2.1 \mu \mathrm{m}$.

Thanks to the diamond-shaped tuning fork [26], the mode sensitive to in-plane accelerations/vibrations is shifted to frequencies larger than $40 \mathrm{kHz}$, so well beyond the acoustic range. This will be later commented in Section V. The overall device area, including the anchor points, is $1600 \mu \mathrm{m} \times 850 \mu \mathrm{m}$.

\section{Tang resonator and drive circuit}

The same module also features a Tang resonator, which is used to generate the single reference frequency for simultaneous off-resonance (or mode-split) operation of the three magnetometers. The resonator structure is implemented as a 3-port configuration (see the top view in Fig. 4a), with motion actuation and detection based on symmetric comb-driven ports, each featuring 113 fingers with a nominal $8 \mu \mathrm{m}$ overlap length and a nominal $2.1 \mu \mathrm{m}$ gap.
The resonance frequency of the first mode (in-plane translational mode, see the FEM of Fig. 4b) is designed at $f_{r}=18.1 \mathrm{kHz}$, in order to operate with a $\Delta f=\left(f_{m}-f_{r}\right)$ $=200 \mathrm{~Hz}$ nominal mismatch with respect to the resonance frequency of the biased magnetometers.

Suspending springs are designed through four 2-fold beams of identical length (details can be seen in Fig. 4c): such first expedient, as described in [17], has the purpose to guarantee that frequency changes under temperature variations are only due to Young's modulus changes rather than to temperature-induced stress effects (details are given in Section V). Further, these beams are designed exactly with the same width as for the beams of the three magnetometers, so that local effects of under or over-etching affect the frequencies of the resonator and of the magnetometers in the same way. This second expedient should ensure a constancy of the frequency difference $\Delta f$ from part to part.

The overall area taken up by the resonator is $290 \mu \mathrm{m} \mathrm{x}$ $840 \mu \mathrm{m}$. The full, ready-to-use module, including the design area, the sealing area and the pad area, can be sized to a single chip with square dimensions of about $(4 \mathrm{~mm})^{2}$. Fig. 5a overlaps a SEM image of the devices to the layout mask of the buried interconnections and of the overall 18 pads.

The resonator is operated within an oscillator loop. The oscillator small-signal loop gain (at the start-up) is larger than one at the expected values of the resonator equivalent resistance (about $30 \mathrm{M} \Omega$ at $6 \mathrm{~V}$ bias voltage applied at the third port - the Tang rotor -, for a resonator quality factor of about 4000). The oscillator circuit, depicted on the left in Fig. $5 \mathrm{~b}$ is based on a transimpedance amplifier front-end (40 $\mathrm{M} \Omega$ feedback resistance), followed by an inverting highgain stage that $(i)$ provides the $-180^{\circ}$ phase shift to satisfy the Barkhausen criterion on the phase, and (ii) leads to saturation, thus providing the nonlinearity that lowers the loop gain down to 1 after the start-up, so to satisfy also the Barkhausen condition on the modulus [27]. A voltage divider is then used to lower the driving voltage waveform to values compatible with the desired resonator motion (about $3 \mu \mathrm{m}$ ). The oscillator does not need amplitude control (AGC [28]), as its goal is solely to provide a reference frequency.

The so obtained oscillating voltage $V_{o s c}$ is then delivered to a Howland current pump (see again Fig. 5b), which injects into the three MEMS magnetometers (electrically connected in series) a current $i_{L}=V_{\text {osc }} / R_{\text {load }}$, well independent of the $1.2 \mathrm{k} \Omega$ resistance caused by the series of the three loops [29]. The end of the current path is directly connected to ground, so that - thanks to the low Al resistance - the MEMS rotors can be initially assumed to be at the ground potential.

\section{E. Sense and demodulation electronics}

As discussed in previous subsections, the Lorentz force determines differential capacitive variations on the magnetometers. For each magnetometer, the sense circuit is thus designed as a low-noise differential capacitive sensing interface, based on a pair of charge amplifiers (CA). Each CA features a feedback capacitance $C_{F}=500 \mathrm{fF}( \pm 20 \%$ 
tolerance) and a feedback resistance $R_{F}=0.6 \mathrm{G} \Omega$ [18]. Their virtual ground is set at $V_{B}=6 \mathrm{~V}$ through the positive input voltage. After a passive, decoupling high-pass filter, the output of each CA is fed to the input of an instrumentation amplifier which turns the measured differential voltage into a single-ended (SE) signal. With respect to preliminary results shown in [1], the circuit is here developed with three parallel channels, to enable simultaneous sensing from the three axes. The possibility to identically replicate the same circuit is only guaranteed by the non-trivial achievement of obtaining well-matched sensitivities for all the devices, along the different sensing axes. This also enables the possibility for the output signals of the three front-end circuits to be sequentially directed, through a multiplexer driven by suitable digital logic, to a single gain, demodulation and filtering chain, implemented using lock-in (LIA) techniques. Multiplexing can occur at a frequency up to $300 \mathrm{~Hz}$, compatible with a system output data rate of $100 \mathrm{~Hz}$ per channel, and thus - according to the sampling theorem - with a maximum theoretical bandwidth of $50 \mathrm{~Hz}$ for the entire system. This bandwidth value is set by a tunable low-pass filter after the demodulation stage. Fig. 5 b reports the complete schematic of the developed drive and sense chains.

The demodulation reference for the LIA is provided by the saturated square wave of the drive circuit described above, so that demodulation occurs at the driving frequency. The LIA output is acquired through a 16-bit analog-to-digital converter, synchronized through a Labview program that manages the whole system operation.

It is worthwhile to remark that only off-resonance operation enables the serial current driving of three magnetometers from a unique reference frequency, and thus a single amplification, filtering and demodulation chain common to all axes. This is rather relevant to save current consumption not only in the devices, but also in the biasing of the electronics stages, in view of an integrated implementation. In this perspective, the possibility to exploit the charge pumps already used for gyroscopes should be considered to provide a DC biasing voltage larger than the nominal supply voltage of the specific CMOS technology.

All the relevant nominal design parameters of the devices (as from Comsol Multiphysics FEM simulations) are given in Table I (reported quality factors represent their measured values; reported resonance frequencies represent their natural, non-tuned values).

\section{SENSITIVITY AND NOISE PREDICTIONS}

\section{A. General considerations about off-resonance operation}

It has been shown in previous works that the motion of a MEMS device characterized by a quality factor $Q$, operated at a frequency $f_{r}$, off-resonance by a quantity $\Delta f=f_{m}-f_{r}$,
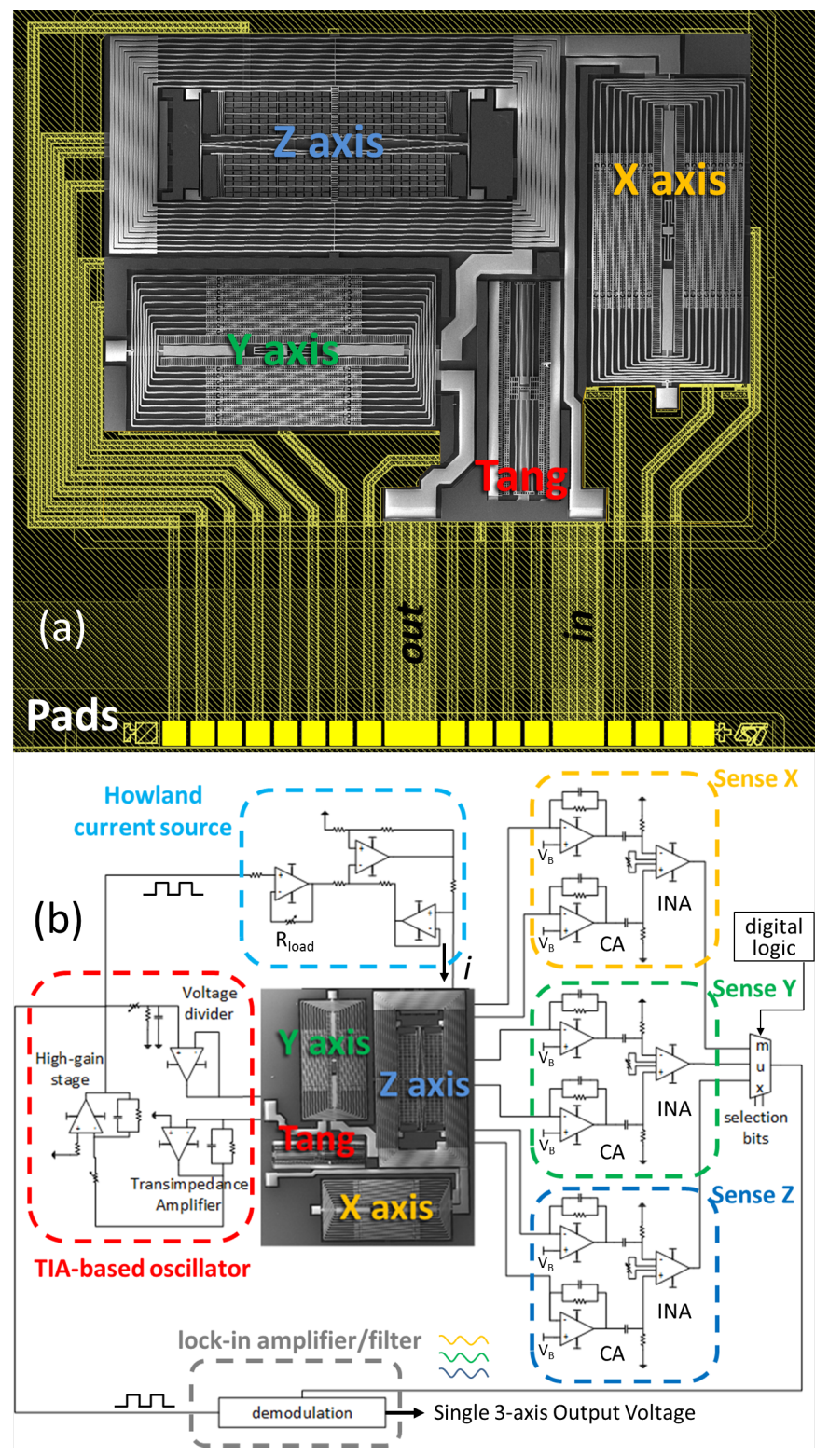

Fig. 5. (a) SEM image of the system, overlapping the interconnections and pads distribution (layout view); (b) block scheme of the developed driving and readout electronics of the system.

which satisfies the following conditions:

$$
\begin{array}{r}
\Delta f \gg \frac{f_{m}}{2 Q} \\
\Delta f \ll f_{m} \\
Q \gg 1
\end{array}
$$

will be amplified with respect to quasi stationary motion by the following quantity [19], named effective quality factor:

$$
Q_{e f f}=\frac{f_{m}}{2 \Delta f}
$$


TABLE I. DEVICE PARAMETERS

\begin{tabular}{|c|c|}
\hline Technology Parameters & Value \\
\hline Process height $(h)$ & $22 \mu \mathrm{m}$ \\
\hline Minimum in-plane gap $\left(g_{h}\right)$ & $2.1 \mu \mathrm{m}$ \\
\hline Minimum vertical gap $\left(g_{v}\right)$ & $1.8 \mu \mathrm{m}$ \\
\hline Al-on-polysi metal width $\left(w_{A l}\right)$ & $3.3 \mu \mathrm{m}$ \\
\hline Al-on-polysi metal thickness $\left(t_{A l}\right)$ & $0.6 \mu \mathrm{m}$ \\
\hline Parameters for IP device & Value \\
\hline Area $\left(A_{t o t}\right)$ & $1300 \times 640 \mu m^{2}$ \\
\hline Natural (untuned) frequency $\left(\overrightarrow{B_{X}}\right.$ mode $)\left(f_{m}\right)$ & $19.25 \mathrm{kHz}$ \\
\hline Effective stiffness $\left(k_{e f f}\right)$ & $83.3 \mathrm{~N} / \mathrm{m}$ \\
\hline Quality factor (Q) & 1060 \\
\hline Natural (untuned) frequency $\left(\overrightarrow{a_{Z}}\right.$ mode) $\left(f_{m, 2}\right)$ & $34.82 \mathrm{kHz}$ \\
\hline Single-ended rest capacitance $\left(C_{0}\right)$ & $487 \mathrm{fF}$ \\
\hline Average Lorentz length $(L)$ & $1100 \mu \mathrm{m}$ \\
\hline Parameters for OOP device & Value \\
\hline Area $\left(A_{t o t}\right)$ & $1600 \times 850 \mu m^{2}$ \\
\hline Natural (untuned) frequency $\left(\overrightarrow{B_{Z}}\right.$ mode) $\left(f_{m}\right)$ & $19.34 \mathrm{kHz}$ \\
\hline Effective stiffness $\left(k_{e f f}\right)$ & $59 \mathrm{~N} / \mathrm{m}$ \\
\hline Quality factor (Q) & 790 \\
\hline Natural (untuned) frequency $\left(\vec{a}_{X}\right.$ mode) $\left(f_{m, 2}\right)$ & $41.77 \mathrm{kHz}$ \\
\hline Single-ended rest capacitance $\left(C_{0}\right)$ & $445 \mathrm{fF}$ \\
\hline Average Lorentz length $(L)$ & $1400 \mu \mathrm{m}$ \\
\hline Parameters for the Tang resonator & Value \\
\hline Area $\left(A_{t o t}\right)$ & $840 \times 290 \mu m^{2}$ \\
\hline Stiffness (k) & $29 \mathrm{~N} / \mathrm{m}$ \\
\hline Quality factor (Q) & 3595 \\
\hline Natural (untuned) frequency $\left(f_{r}\right)$ & $18.1 \mathrm{kHz}$ \\
\hline
\end{tabular}

It is for instance the case of the sense mode of gyroscopes operated in mode-split conditions [13]. Well-known advantages of this kind of MEMS operation are:

- solution of the bandwidth vs thermomechanical noise density trade-off (in resonant operation the former worsens, the latter improves when lowering the damping coefficient);

- tolerance to changes of the quality factor $Q$ with respect to environmental changes (temperature [30], aging, or from part to part): indeed from Eq. 2 one clearly see that $Q_{\text {eff }}$ shows no dependence on $Q$;

Specifically, the operation of MEMS magnetometers under off-resonance conditions was first proposed in [18], carrying the following, further advantages for the proposed 3-axis implementation:

- avoidance of the challenge to provide three reference frequencies for the Lorentz current which accurately match and track the three magnetometers frequency;

- possibility to make the same current re-circulate through the three devices. This concept is experimented in details for the first time in this paper, following the prediction in [1]. This would be possible for resonant operation at high $\mathrm{Q}$ only in the ideal (and rather unrealistic) case where the three devices have perfectly and stably matched frequencies, one another and with the reference;
Further, the use of a MEMS frequency reference in the same die of the magnetometers, as proposed in this work, adds this final advantage:

- high-tolerance to changes of the resonance frequency with temperature: indeed, with the proposed solution, a self-tracking between the reference and the magnetometers frequency changes versus temperature is achieved, so to keep the scale-factor well stable. This concept, first anticipated in [19], was recently verified in [31] for a single-axis structure using two MEMS from separate chips. This paper verifies the constancy of the $\Delta f$ term for the proposed single-chip, 3-axis system, through temperature measurements reported in Section V.

The disadvantage to pay is that the transduction action from magnetic field change into displacement is lower by a ratio $Q_{\text {eff }} / Q$, e.g. a factor $1 / 20$ for a typical $Q=1000$ and a typical $Q_{e f f}=50$. There are no consequences of this reduction in terms of input-referred thermo-mechanical noise: indeed both the signal and this type of noise are transduced by the same scale-factor. There may be on the contrary consequences on the impact of electronic noise: indeed the same output noise density, divided by a lower transduction factor, turns into a larger input-referred rms magnetic field. These considerations are briefly formalized through equations in the next subsections.

\section{B. Sensitivity calculation}

Consider a differential capacitive magnetometer like those presented in Section II, with a nominal resonance frequency $f_{m}$, driven off-resonance by a quantity $\Delta f$ through an $\mathrm{AC}$ current with peak amplitude $i$. The single-ended parallel-plate capacitance is $C_{0}$ and the gap between plates is $g$. One can write the scale-factor (or sensitivity) in terms of system output voltage variation $\delta V_{\text {out }}$ per unit magnetic field change $\delta B$ :

$$
\frac{\delta V_{\text {out }}}{\delta B}=\frac{\delta F_{L}}{\delta B} \frac{\delta x}{\delta F_{L}} \frac{\delta C}{\delta x} \frac{\delta V_{\text {out }}}{\delta C}
$$

Considering that the displacement along the sensing direction $x$, caused by the AC Lorentz force $F_{L}=B \cdot i \cdot L \cdot N_{\text {loop }}(L$ being the average spring length and $N_{\text {loop }}$ being the number of re-circulation paths), is amplified by $Q_{e f f}$, one can derive the sensitivity expression per unit current consumption:

$$
\frac{\delta V_{\text {out }}}{\delta B \cdot i}=N_{\text {loop }} L \frac{Q_{\text {eff }}}{k_{\text {eff }}} \cdot 2 \frac{C_{0}}{g} \frac{V_{B}}{C_{F}} G_{\text {eln }}
$$

In the equation above, $k_{e f f}$ is the effective device stiffness in units of $N / m$. For a Y-axis device, this corresponds (for small tilting angles) to the torsional stiffness divided by the square of the average torque arm, $d$ (see Fig. 2a). For a Z-axis magnetometer like the one shown in Fig. 3, it corresponds to the stiffness of one half of the device (anti-phase motion), pre-multiplied by a factor 2 due to the distribution of the Lorentz force across the springs. $G_{\text {eln }}$ represents the gain of all electronic stages beyond the CA.

With the nominal parameters given in Table I, one can calculate the nominal sensitivity per unit Lorentz current for a 200 
$\mathrm{Hz}$ mode-split to be $\frac{\delta V_{\text {out }}}{\delta B \cdot i}=5.56 \mu \mathrm{V} /\left(\mu T \mu A_{\text {rms }}\right)$, nominally identical for the proposed IP and OOP devices, and independent of the value of the quality factor.

\section{Noise density calculation}

Main noise contributions for the proposed system are given by the Brownian force noise power spectral density $\left(S_{n B}\right.$, in units of $\left.N^{2} / H z\right)$, by the Johnson noise power spectral density of the feedback resistance $\left(S_{n R}\right.$, in units of $\left.A^{2} / H z\right)$, and by the operational amplifier voltage noise power spectral density $\left(S_{n V}\right.$, in units of $\left.V^{2} / H z\right)$. All the contributions can be conveniently written in terms of input-referred equivalent rms magnetic field density $\sqrt{S_{i n}}$ (in units of $T_{r m s} / \sqrt{H z}$ ). For Brownian noise at a temperature T, $k_{B}$ being the Boltzmann constant, one can find:

$$
\sqrt{S_{\text {in }, B}}=\frac{2}{N_{\text {loop }} i L} \sqrt{4 k_{B} T b}
$$

(the factor 2 arises because noise, dominated by interactions with surrounding gas particles in squeezed-film regions, acts directly on the suspended frame, and not distributed along the springs like the Lorentz force does).

The two feedback resistors noise is brought back to the system input through the MEMS capacitive gain at a frequency $\omega_{r}=$ $2 \pi f_{r}$ :

$$
\sqrt{S_{\text {in }, R}}=\frac{1}{N_{\text {loop }} i L} \sqrt{\frac{8 k_{B} T}{R_{F}}} \frac{g \cdot k_{\text {eff }}}{C_{0} V_{B} Q_{\text {eff }} \cdot \omega_{r}}
$$

Finally the amplifiers noise can be written by considering its amplification to the system output through the parasitic capacitance, and by referring it to the input through the sensitivity:

$$
\sqrt{S_{\text {in }, V}}=\frac{1}{N_{\text {loop }} i L} \sqrt{2 S_{n V}} \frac{C_{P}}{V_{B}} \frac{g \cdot k_{e f f}}{C_{0} Q_{e f f}}
$$

It is relevant to note that adding re-circulation loops improves all of the shown noise contributions: this differentiates the calculations shown here from previous works not based on such current reuse.

With the parameters given in Table $\mathrm{I}$, at a $6 \mathrm{~V}$ biasing voltage and assuming a $5 \mathrm{pF}$ parasitic capacitance $C_{P}$, an overall input-referred theoretical noise density per unit current consumption of $13.5 \mu T \mu A_{r m s} / \sqrt{H z}$ and $19.2 \mu T \mu A_{r m s} / \sqrt{H z}$ is obtained for OOP and IP devices, respectively. This means that values as low as sub-200 $n T / \sqrt{H z}$ can be theoretically obtained for Lorentz current values of $100 \mu A_{r m s}$ only. Noise continues to be dominated by the Brownian contribution up to parasitic capacitances in the order of $15 \mathrm{pF}$, for an operational amplifier input noise density $\sqrt{S_{n V}}=4 \mathrm{nV} / \sqrt{\mathrm{Hz}}$ (ADA4817 from Analog Devices).

The system is on the whole compatible with low-power electronics based e.g. on the integrated readout presented in [23]. More in general, from an integrated circuit perspective, using only $100 \mu A_{r m s}$ leaves as much as $150 \mu A_{r m s}$ for the electronics to be competitive with state-of-the-art overall current consumption in the order of $250 \mu A_{r m s}$ [32].

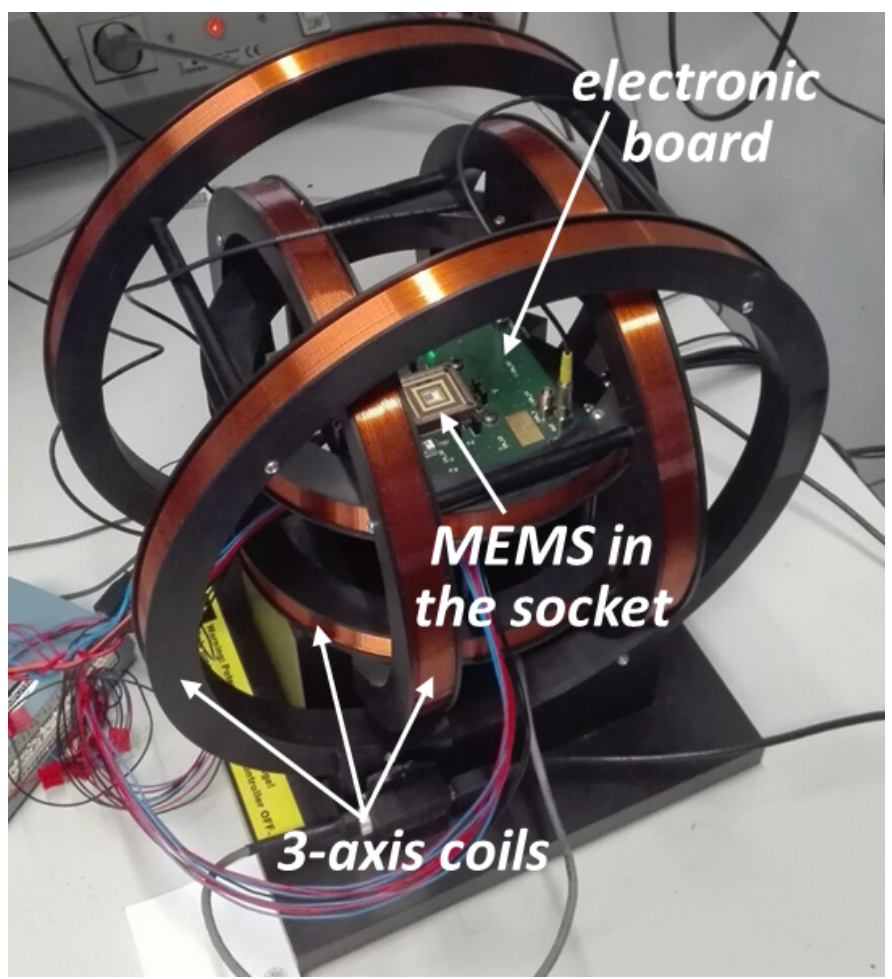

Fig. 6. Picture of the setup, with the MEMS mounted on a carrier, plugged into a socket on the driving/readout board within the Helmholtz coils setup.

\section{SENSITIVITY AND NOISE CHARACTERIZATION}

Experimental characterization is performed using a 3 -axis Helmholtz coil setup from Micromagnetics Inc., which accommodates the readout board as shown in Fig. 6. The setup provides a field generation accuracy of $300 \mathrm{nT}$, with maximum applicable fields in the order of $5.5 \mathrm{mT}$ per axis.

\section{A. Sensitivity}

Two types of sensitivity characterization are performed. The first one is obtained by sweeping the magnetic field from -5 $\mathrm{mT}$ to $+5 \mathrm{mT}$ along every axis, one at a time. The choice of this range is motivated by a continuous demand of extended FSR in consumer IMUs (see e.g. [32]). The results are reported in Fig. 7, where (a) shows a measured scale-factor of 5.8 $\mu \mathrm{V} /\left(\mu T \mu A_{r m s}\right)$ for the $\mathrm{Z}$-axis device and a cross-axis rejection of $33 \mathrm{~dB}$ and $42 \mathrm{~dB}$ for the other two axis under Z-axis fields (limits may be due to manual alignment). Similarly, (b) and (c) show sensitivities for the twin Y-and $\mathrm{X}$-axis devices in the order of $6.4 \mu \mathrm{V} /\left(\mu T \mu A_{r m s}\right)$. This value is slightly larger than predictions: a possible motivation is in an underestimation of the vertical capacitance between the rotational frame and the underneath stators (see Fig. 2c), in particular when accounting the effect of perforating holes. The matching between the sensitivities along the three sensing axes is anyway quite good, and the cross-axis rejection for IP devices is always larger than $39 \mathrm{~dB}$. The linearity error, calculated as a percentage of the FSR assumed as $5 \mathrm{mT}$, is lower than $0.2 \%$ along the tested 
magnetic field range.

The second type of sensitivity characterization consists in generating a sphere through a magnetic field vector of constant amplitude which rotates in the 3-D space quasi-stationarily in time. This is repeated for different field modulus values. One purpose is to verify that the FSR of the proposed MEMS magnetometers shows no intrinsic limits related to cross-axis effects. This is on the contrary a typical limitation in devices based on magnetic materials: e.g. the FSR of a given axis of an AMR device is not limited by its linearity errors but it is rather limited by the flipping mechanism occurring when a field along an orthogonal direction occurs [33]. Fig. 7d shows the obtained result for spheres having a growing field radius up to $5.5 \mathrm{mT}$. The reader can note that, after correcting for the small sensitivity differences in the digital domain, the spherical shape is well kept up to the maximum applicable field.

The result thus also verifies a second purpose: the immunity of the developed system to coupling/cross-talk of electri$\mathrm{cal} /$ mechanical signals between different channels. Such a thing could in principle occur as all the devices and channels operate at an identical frequency.

\section{B. Bandwidth}

In general, the bandwidth in off-resonance operation can be set up to large fractions of the frequency difference $\Delta f$. The required precaution is to avoid that the response peak occurring for an AC field frequency exactly matching the value $\Delta f$ (about $200 \mathrm{~Hz}$ in our system) is amplified by the quality factor peak. This goal is achieved by using a filter at the LIA output whose pole has a low-pass frequency of $50 \mathrm{~Hz}$.

Fig. 8 reports the obtained results for all the devices when sweeping the field between $0.5 \mathrm{~Hz}$ to $500 \mathrm{~Hz}$. The reader can note that the $-3 \mathrm{~dB}$ value is the same for all the sensing axes, and that the peaks at about $200 \mathrm{~Hz}$ are well filtered below the $-3 \mathrm{~dB}$ value.

\section{Noise}

Noise characterization is performed by measuring the Allan deviation while the Lorentz current is serially injected into the three devices, biased as in operating conditions. The results, reported in Fig. 9, demonstrate a white noise density per unit current consumption of $20.3 \mu T \mu A_{r m s} / \sqrt{H z}$ for the Z-axis device, including the electronic noise contribution. This value is in line with the predictions, if one considers a parasitic capacitance of $17 \mathrm{pF}$, a value which is compatible with the used CLCC68 carrier/socket combination. This is confirmed by the fact that noise for the X-and Y-axis devices is quite similar to the Z-axis device, just improved to $18.5 \mu T \mu A_{r m s} / \sqrt{H z}$ by the sensitivity difference. The matching between noise performance along all the axes is quite good.

For an input current of $100 \mu A_{r m s}$, a $70 \mathrm{nT}$ stability is reached at about $30 \mathrm{~s}$ for both IP devices. For the Z-axis device, stability is poorer by roughly a factor $2(160 \mathrm{nT})$ at about half the observation time. Hypothesis to explain this finding will be drawn in the following Section.
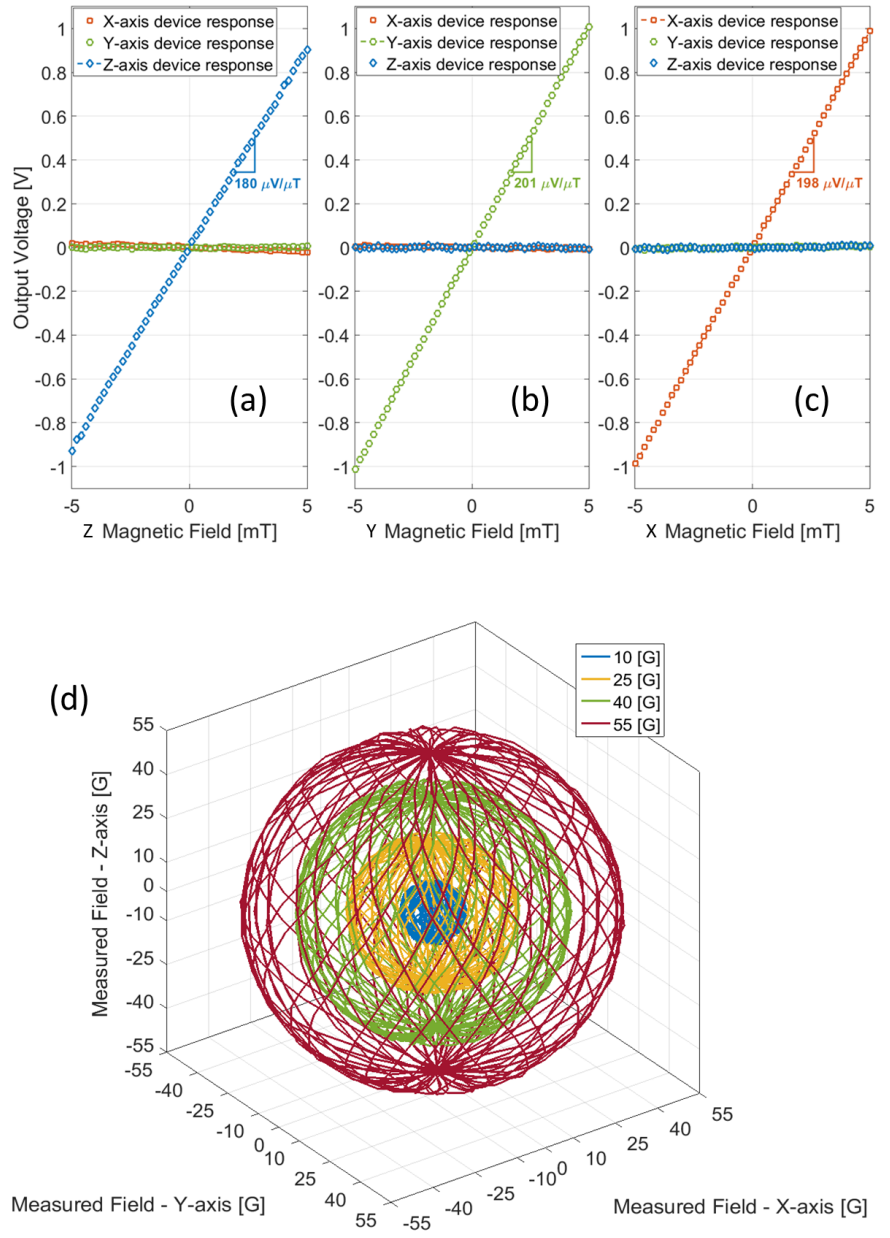

Fig. 7. Output voltage of the three different sensing channels versus an input magnetic field applied along (a) the Z-axis, (b) the Y-axis and (c) the $\mathrm{X}$-axis. The spheres of different 3-D magnetic field modulus in (d) are captured by the system while slowly sweeping the tip of the magnetic field vector in space, so to describe a spherical path.

\section{STABILITY UNDER ACCELERATIONS AND TEMPERATURE CHANGES}

\section{A. Rejection of accelerations}

This subsection verifies, for the first time on this type of system, immunity to DC and AC accelerations acting on the magnetometers. In order to highlight the harmfulness of accelerations, in the particular case of off-resonance operation, a numerical example is first given. One can consider the effect of $a=1 \bar{g}$ acceleration on a device resonating, like in this work, at about $18 \mathrm{kHz}$. The corresponding displacement $x_{a c c}$ under such an acceleration is easily derived from the equation below:

$$
x_{a c c}=\frac{a}{\omega_{m}^{2}}=\frac{a}{\left(2 \pi f_{m}\right)^{2}}=0.75 \mathrm{~nm}
$$

On the other side, one can check the displacement $x_{m a g}$ occurring when measuring a $100 \mathrm{nT}$ rms magnetic field change at $100 \mu A_{r m s}$ driving current, i.e. the best stability situation 


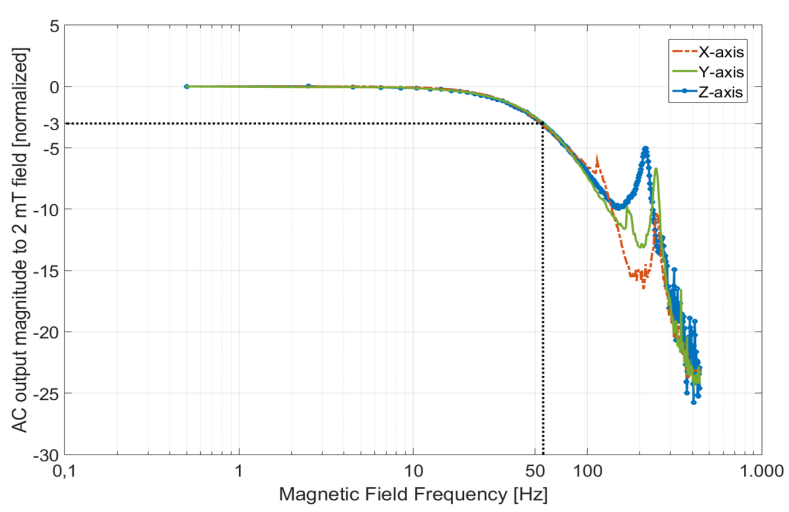

Fig. 8. Measured system bandwidth for all the magnetometers of this work. Note the $-3 \mathrm{~dB}$ value matching about $50 \mathrm{~Hz}$ for all the devices. Beyond 200 $\mathrm{Hz}$, the accuracy in the field generation by the Helmholtz coils setup degrades differently for the different axis (due to their different diameter, see Fig. 6, which explains the apparently different behavior for X-and Y-axis devices.

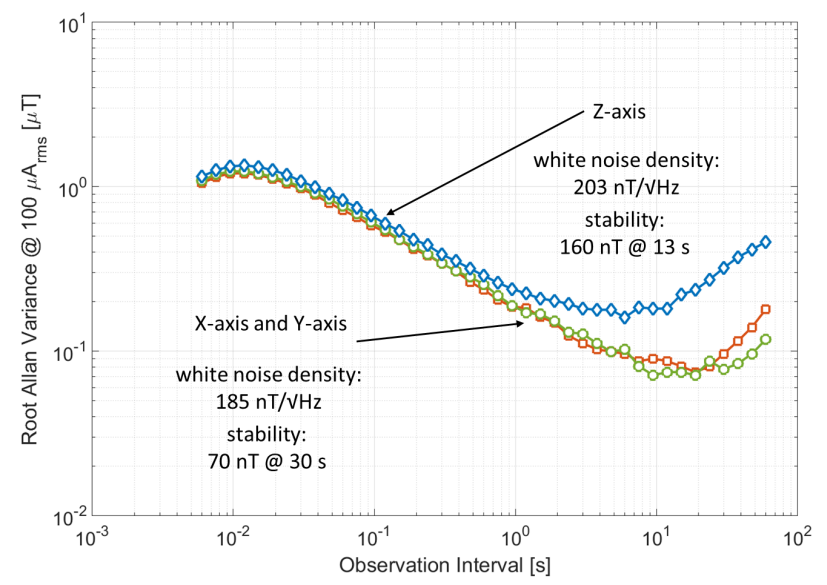

Fig. 9. Allan variance for the magnetic field density, for the three devices of this work. Results are obtained at a $100 \mu A_{r m s}$ AC driving current.

obtained in the Allan variance graph of Fig. 9. Considering the spring length and the stiffness value of the proposed devices, and off-resonance operation with loop recirculation, one gets:

$$
x_{\text {mag }}=\frac{B \cdot i \cdot N_{\text {loop }} L \cdot Q_{\text {eff }}}{2 \cdot k_{\text {eff }}}=75 \mathrm{fm}
$$

There are therefore 4 orders of magnitude between the displacement caused by an (even small) acceleration and the target field to measure. Even if vibrations usually occur at frequencies lower than the modulation frequency $f_{m}$, and even in presence of good electronic filtering, canceling out such a huge difference remains challenging. The fact that accelerations represent a critical issue is, by the way, testified also by typical gyroscopes architectures based on tuning forks: it is indeed a parallel situation, where forces to be sensed are very small compared to those caused by accelerations.

The strategy here adopted to improve robustness against these effects is the use (for all the axes) of an architecture that implements a capacitive sensing configuration which inherently rejects accelerations as a common mode, differing from most of previous works, especially for Z-axis devices [2], [4], [14], [18], [19], [21], [34]. The strategy is further completed by the shift of the mode sensitive to accelerations to high frequencies, as discussed below.

In particular, for devices sensitive along the $\mathrm{X}$ or $\mathrm{Y}$ axis:

- accelerations along the $\mathrm{Z}$ direction (orthogonal to the substrate surface) are the most critical ones. Their effect is minimized because the structure is balanced around the mass center, as previously proposed e.g. in [35]: this implies that an acceleration does not excite the first, differential mode but a high-order mode. This wing-like, in-phase torsional mode (see Fig. 10a) occurs at about $32 \mathrm{kHz}$, as experimentally demonstrated through an electromechanical characterization of the modes shown in Fig. 10b [29]. Upward shifting the acceleration sensitive mode by a factor $\approx 2$ turns into $\mathrm{a} \approx 4$-fold larger immunity as suggested by Eq. 8. Besides, the excitation of such a mode gives rise to a commonmode capacitance variation, inherently rejected by the differential readout;

- the effects of accelerations along the X direction, parallel to the torsional beams, are minimum as the device is very stiff along this direction;

- accelerations along the other in-plane (Y) direction give rise to lateral in-plane displacements. The corresponding mode falls at about $43 \mathrm{kHz}$. Thanks to a $5 \mu \mathrm{m}$ enclosure distance of the bottom electrodes mask within the frame mask, such lateral motion does not cause capacitance variations, as the electrodes facing area does not change.

For devices sensitive to the $\mathrm{Z}$ axis:

- accelerations along the $\mathrm{X}$ direction, parallel to the substrate surface, are the most critical ones for this device. Their effect is minimized by design thanks to the diamond-shaped geometry of the tuning fork, with clamped-clamped beams at its ends. This geometry is known to shift the in-phase mode to a frequency larger than the anti-phase mode [26]. This is predicted by FEM simulations (see Fig. 11a) and experimentally verified in the modes electromechanical characterization (Fig. 11b). The upward frequency shift, in this case, gives a 5.5-fold larger immunity to accelerations than in a situation (like all those referenced above) where the mode sensitive to accelerations is the same as for the magnetic field. Once more, for the proposed geometry the effects of such a motion is a common mode, rejected in the differential readout;

- effects of accelerations along the other in-plane direction (Y), as well as of vertical (Z-axis) accelerations, are minimum as the device is very stiff along both these axes.

To proof the goodness of the design in terms of acceleration rejection, the sensitivity measurements described in Section IV were repeated under different conditions of accelerations. These include $1 \bar{g}$ (DC) along the Z-direction (repeated 6 times), $\pm 1 \bar{g}$ (DC) along the X-direction (repeated 3 times 


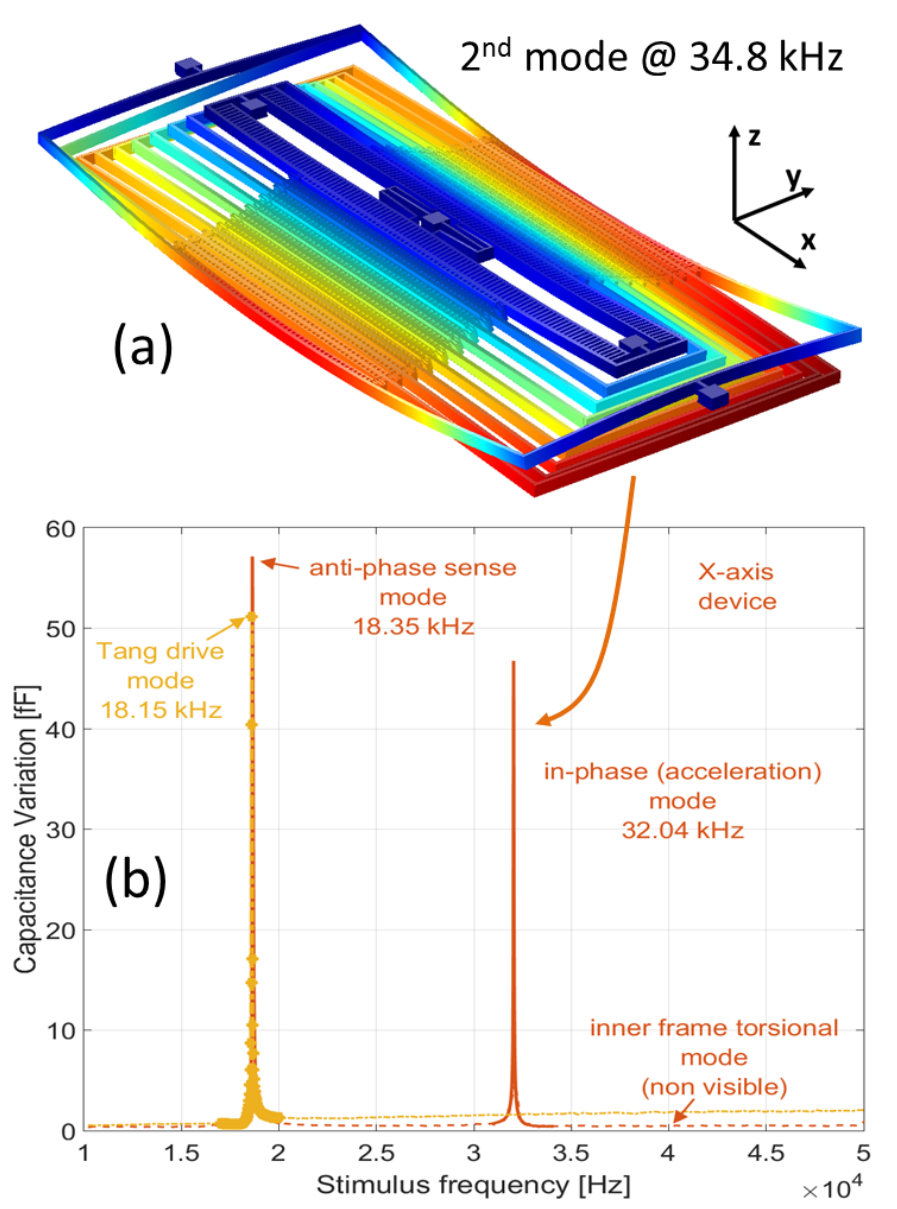

Fig. 10. (a) FEM predictions of the second mode (the one excited by accelerations) of the IP devices, and experimental electromechanical verification of its frequency (b). The measured response of the Tang resonator is also shown in markers for sake of completeness.

each), $\pm 1 \bar{g}$ (DC) along the Y-direction (repeated 3 times each), and $\pm 3 \bar{g}$ AC random-direction accelerations (repeated 3 times). To obtain these different accelerations while measuring the sensitivity, the whole setup was tilted or subject to shakes and shocks. The acceleration ground truth was obtained through a reference accelerometer rigidly mounted on the setup. The frequency of the applied AC accelerations is limited to $<10 \mathrm{~Hz}$ due to the bulky setup (Fig. 12a).

Fig. 12b reports a summary of the results: every column corresponds to a different type of applied acceleration, and every row to a different type of device. In each graph, the percentage deviation of each measurement with respect to the average sensitivity measurement from all these collected data is plotted versus the applied magnetic field. The number of curves in each graph corresponds thus to the number of repetitions of the measurement under that specific acceleration. One can note that deviations are always lower than $1 \%$, thus indicating a good tolerance of the whole magnetic field sensing unit to such actions.

No significant offset change was found while tilting the device:

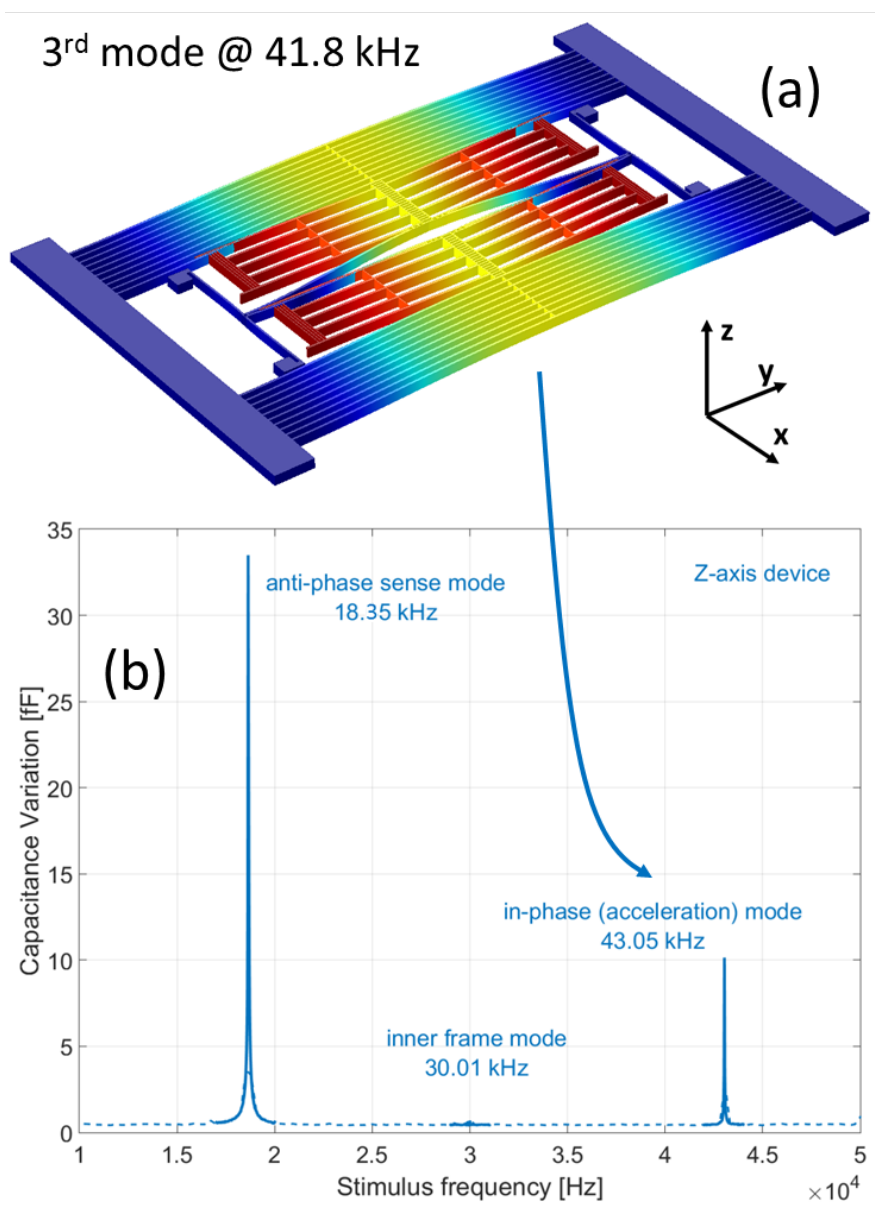

Fig. 11. (a) FEM predictions of the second device mode (the one excited by accelerations) of the OOP device, and experimental electromechanical verification of its frequency (b).

the DC value (for no applied field from the Helmholtz coils) changed by few to few tens $\mu \mathrm{T}$ according to the change in the orientation with respect to Earth magnetic field.

\section{B. Temperature stability of the modes difference}

One characteristic in off-resonance operation is to have a scale-factor linearly dependent on $Q_{e f f}$ in Eq. 2. This subsection reports for the first time a brief theoretical prediction and experimental measurements on the stability of $Q_{\text {eff }}$ versus temperature changes for a 3-axis magnetometer which embeds in the same die a MEMS resonator as the frequency reference. Similar measurements, as proposed in [36], were recently shown in [31] for a single axis device using separate dies for the sensor and the frequency reference.

Given the temperature coefficient of frequency for the magnetometer, $T C f_{m}$, and for the resonator, $T C f_{r}$, one can write the effective quality factor dependence on temperature variation $\Delta T=T-T_{0}$, with respect to a reference temperature 

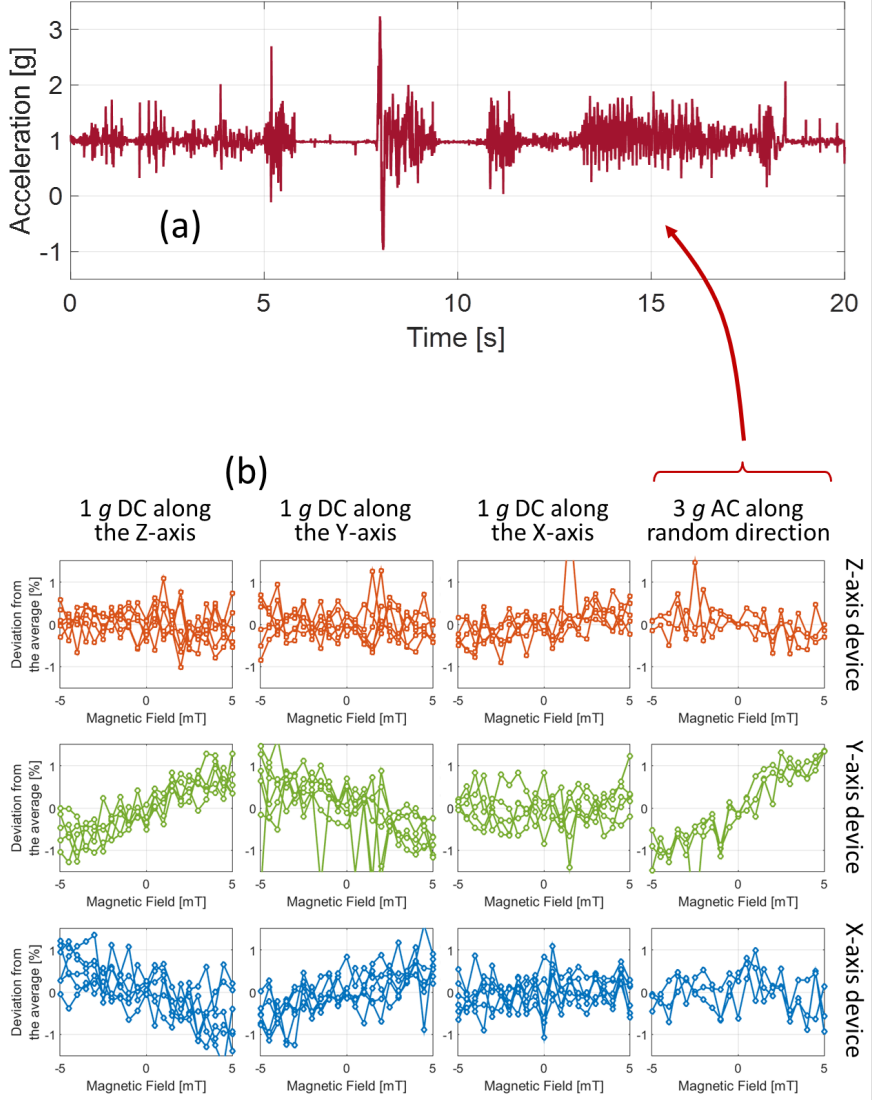

Fig. 12. (a) example of applied AC acceleration modulus profile; (b) deviations of the measured sensitivity from the average value, when operating measurements under DC and AC accelerations as indicated at the top of each column. Each row corresponds to a different device, as indicated to the right. For all the measurements, a sensitivity change lower than $1 \%$ is observed.

$T_{0}$ :

$$
Q_{e f f}=\frac{1}{2} \frac{f_{m 0}\left[1+T C f_{m} \Delta T\right]}{f_{m 0}\left(1+T C f_{m} \Delta T\right)-f_{r 0}\left(1+T C f_{r} \Delta T\right)}
$$

There are two major sources of frequency variation against temperature in MEMS, related to the material and to the geometry respectively. The former is the unavoidable dependence of polysilicon Young modulus on $\mathrm{T}$, with a $T C f$ of about -30 $\mathrm{ppm} / \mathrm{K}$. The latter is related to the possible presence of residual stress after the fabrication process, whose value changes with temperature: in this case, the $T C f$ value depends on whether the specific geometry allows a relief of the residual stress on the springs or not.

In the optimum situation, the second term is made negligible for the resonator and the magnetometers: the temperature drift of all the frequencies involved in the operation of the 3axis magnetometer shows in this case the same coefficient $T C f_{0}=T C f_{m}=T C f_{r}$ :

$$
Q_{e f f}=\frac{f_{m 0}\left(1+T C f_{0} \Delta T\right)}{2\left(f_{m 0}-f_{r 0}\right)\left(1+T C f_{0} \Delta T\right)}=\frac{1}{2} \frac{f_{m 0}}{f_{m 0}-f_{r 0}}
$$

An identical $T C f$ for $f_{r}$ and $f_{m}$ indicates stability in the

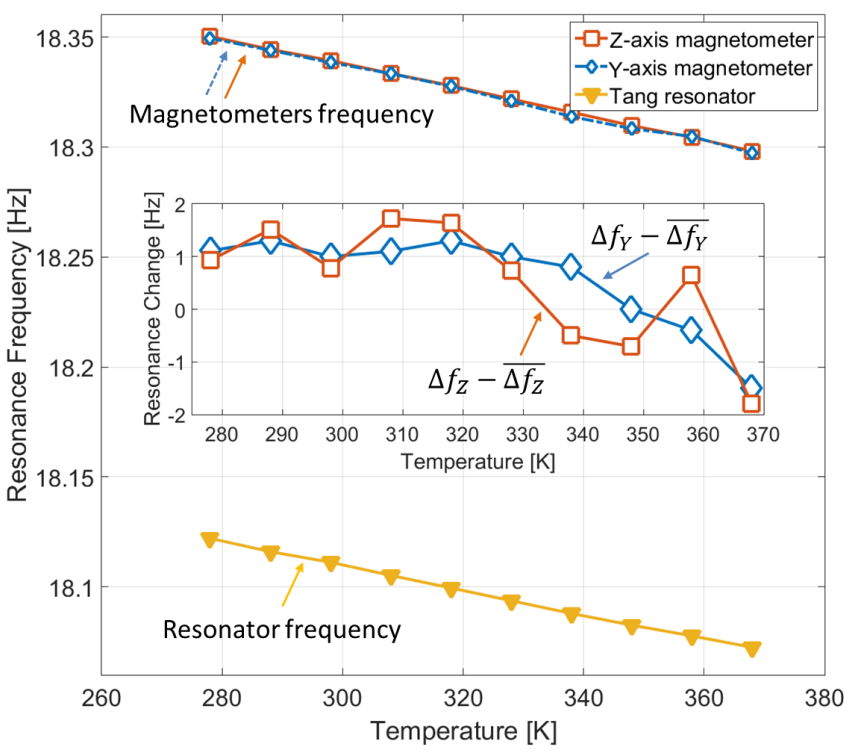

Fig. 13. Frequency behavior vs temperature for the magnetometers and the Tang resonator used in this work. The TCf for X-and Y-axis sensors perfectly mathces the resonator one. The TCf for the Z-axis device well matches the resonator one after linear compensation. The inset indicates the frequency difference between magnetometers and resonator modes (i.e. the variation in the $\Delta f$ term) versus temperature.

$Q_{e f f}$, and in turn in the sensitivity versus temperature.

To verify this, the magnetometer frequencies were measured through an electromechanical characterization platform [37], with the 3 -axis module kept inside a climatic chamber. The temperature was swept between $5^{\circ} \mathrm{C}$ and $95^{\circ} \mathrm{C}$, by $10^{\circ} \mathrm{C}$ steps. Figure 13 reports the measured results, where the frequency of the Y-axis magnetometer (square markers) and of the Tang resonator (triangle markers) show a temperature coefficient of frequency (TCf) of $-0.55 \mathrm{~Hz} / \mathrm{K}$. The overall variation in the $\Delta f$, shown in the inset, turns out to be in the order of \pm 1 $\mathrm{Hz}$ in a range of $90 \mathrm{~K}$, corresponding to about $55 \mathrm{ppm} / \mathrm{K}$ at a mode-split value of $200 \mathrm{~Hz}$. The frequency of the $\mathrm{Z}$-axis magnetometer showed $\mathrm{a} \approx 30 \%$ larger TCf: this is probably caused by the fact that the springs, though rather long, are clamped on both sides without chances of stress-relief. As shown in Fig. 13 however, a simple linear correction (applied to the scale-factor in the digital domain) brings the values (diamond markers) quite close to what obtained for the $\mathrm{X}$ and Y-axis devices.

These measurements confirm the effectiveness of the proposed solution of using a MEMS resonator to provide the reference harmonic for the Lorentz current in off-resonance operation.

\section{Offset sources and compensation}

In several MEMS, e.g. in inertial sensors, offsets can easily reach values comparable to or larger than the FSR. It is the case of accelerometers [38], due to the combination of process stresses and inherently low elastic stiffness. It is the case of 
gyroscopes, where quadrature error can be one or two orders of magnitude larger than the FSR. It is also the case of the magnetometers of this paper, due to the following two major offset sources.

The first one is common to other MEMS magnetometers. It is the undesired capacitive AC coupling between the drive signal and the sense channels. In principle, if the coupling is symmetric into both the differential channels, it should be canceled in the differential readout. However, small, unavoidable asymmetries (e.g. due to the crowded interconnections and the impossibility to make all of them symmetric, see again Fig. 5a) make an offset signal appear at the output of each device. This kind of offset can be as large as the FSR (few mT), but it is not expected to significantly drift with environmental changes, as the coupling capacitances should be stable with temperature. The second source of offset is peculiar to the multi-loop structure. It is caused by the combination of an electrical and a mechanical nonideality of each magnetometer:

- electrical: the rotor voltage was assumed so far to be at the ground potential. However, the Al path has its own finite resistance, though small. This causes a residual small AC voltage (in the order of $<1 \mathrm{mV}$ ) appear on the rotor. If the stators were perfectly symmetric with respect to the rotor, this voltage would be applied identically to the two sensing capacitances, thus resulting in a common-mode voltage;

- mechanical: yet, the presence of an unavoidable, native, mechanical offset determines a variation in the rest gap of the differential capacitances, and thus results in an offset at the output. From analytical models and typical mechanical offset values, this contribution is expected to be smaller than the former one by roughly an order of magnitude. However, it is expected to vary with temperature, as in general the mechanical offset, caused e.g. by pre-stresses in the springs, is itself a function of temperature.

Effectively, an offset in the order of few mT, likely due to a combination of the aforementioned origins, was measured on the devices when applying an identical $6 \mathrm{~V}$ bias value to all the sensing stators.

Compensation of such offsets is needed at a rough level to avoid saturation, at a higher level to guarantee the possibility to fully exploit the whole electronic dynamic range, and at the highest level (yet, not pursued in this paper) to compensate also possible offset drifts.

Options for offset compensation in MEMS are in general split into two categories: electronic compensation and electromechanical compensation. The former is usually based on the injection of a signal equal and opposite to the offset at a certain point in the electronic chain, before signal saturation (see e.g. [39]). It requires added electronic stages and an initial calibration. On the contrary, the latter generally compensates mechanical anomalies (see e.g. the well-known Tatar compensation scheme in gyroscopes, [40]).

There could be actually a third option for offset compensation, which is chopping of the stators voltage. This kind of

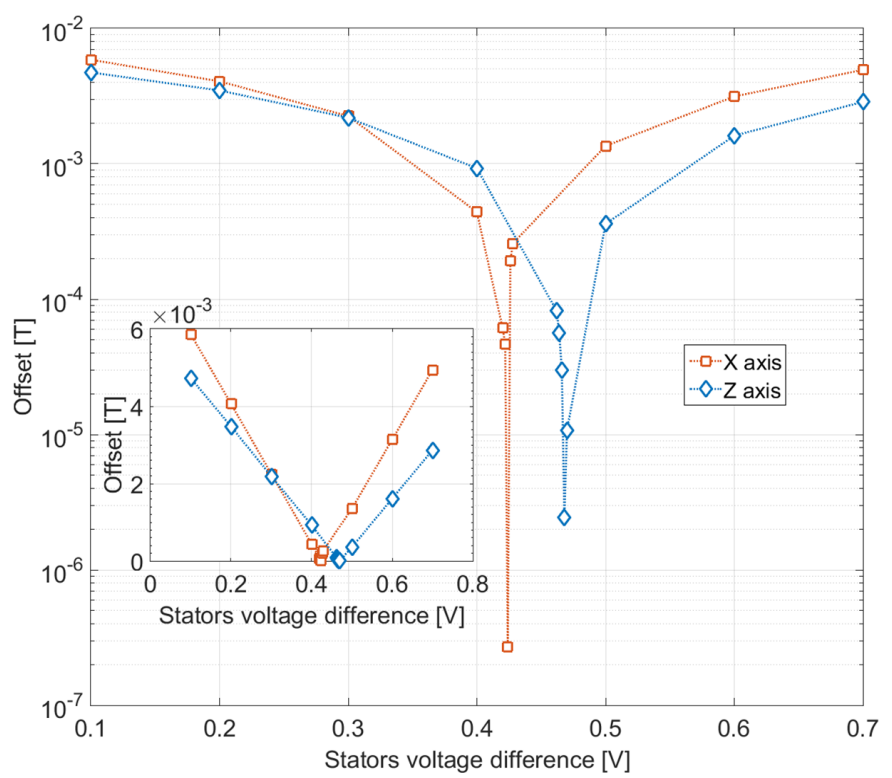

Fig. 14. Offset compensation of IP and OOP devices via the application of a small DC voltage difference at the virtual ground of the two charge amplifiers. The compensation is linear with the applied difference, as shown by the inset, and reaches values down to the $\mu \mathrm{T}$ range, as shown in log-scale in the whole figure.

compensation would also probably have beneficial effects on offset drifts. It was however not implemented in this work and it is therefore not discussed, as it would require one further modulation and one further demodulation block, leading to increased power consumption, incompatible with dissipation targets in view of an integrated electronic implementation.

The used offset compensation technique for the magnetometers in this manuscript is of the electromechanical type. The choice has been done to avoid adding extra power consumption to the electronic chain. Compensation was obtained via the application of small DC voltage differences at the two stators of each magnetometer. This electromechanical action sets the suspended mass in a position such that the differences in the capacitive gains of the two stators compensate the differences in crosstalk (a similar approach was also proposed in [41]). Fig. 14 shows how the initial offset, in the order of 5-6 mT for both IP and OOP devices, is trimmed down to the $\mu \mathrm{T}$ range, through a difference in the applied voltage between differential stators in the order of $\pm 0.2 \mathrm{~V}$. As a consequence of the achieved compensation, the electronic FSR is now fully matched with the magnetic field sensing range.

Note that this approach just compensates the offset without eliminating its source at the origin. This means that offset drifts - e.g. with temperature - will not be compensated via this technique. Looking back at the Allan measurements previously shown in Fig. 9, observed instability and drifts in uncontrolled laboratory environment can be likely ascribed to this issue. In future works, temperature compensation methods, applied as digital processing, could be exploited to improve stability, as often implemented on inertial MEMS available on the market. 


\section{CONCLUSIONS}

The paper presented a complete sensing unit for magnetic field detection along three axis, fabricated in a currently available industrial MEMS process. The unit is based on the Lorentz force principle, exploited in off-resonance mode using a reference resonant element designed within the same chip of the magnetometers. The complete characterization verifies the sensitivity predictions, and demonstrates its immunity to $\mathrm{AC}$ and DC accelerations. Further, it shows a $185 \mathrm{nT} / \sqrt{\mathrm{Hz}}$ noise density at $100 \mu A_{r m s}$ current consumption (equivalent to about $6.6 \mu T \mu A_{r m s} / \sqrt{H z}$ per axis). Along the \pm 5.5 $\mathrm{mT}$ FSR, the linearity error and the cross-axis rejection are compatible with typical requirements of consumer applications. Scale-factor dependence on the frequency mismatch in off-resonance operation is lower than $55 \mathrm{ppm} / \mathrm{K}$, assessed via the measurement of the TCf of the embedded resonator and of the magnetometers.

A comparison against commercially available products and recent research achievements is reported in Table 2. As done in previous works on magnetometers, the considered figure of merit (FOM) is noise density per unit bandwidth and unit current consumption. This FOM accounts for the fact that the same magnetometer can obviously reach higher sensitivity and lower noise by using a larger current, so it is a fair approach to normalize to the driving current. Together, also the maximum achievable bandwidth is used as a second comparative term: this accounts for the fact that resonant magnetometers can reach large scale factor, but at the cost of a much reduced sensing bandwidth, generally incompatible with target applications. Further, the FSR is used as a third comparative term: this accounts for the fact that several magnetometers based on other technologies, including magnetic materials, suffer from inherent FSR limitations due to magnetic domain flipping. Increasing the FSR is one of the most demanded parameter evolution for magnetometers, but at the same time one of the toughest challenges e.g. for AMR magnetometers developers. Finally, the footprint has obviously a relevance when miniaturization is pursued, and it is thus included in the comparative Table.

Overall, the proposed system reaches the best FOM within MEMS devices targeting consumer applications. This FOM is even better than several commercially available devices based on other technologies, with potential advantages of having (i) a larger FSR, (ii) a programmable FSR (changing the driving current), (iii) compatibility with other inertial sensors to form single-process 9-axis IMUs.

The overall estimated footprint for such a device is in the order of $4 \times 4 \mathrm{~mm}^{2}$, i.e. four times larger than smallest commercially available products. This may appear as one limiting factor for the proposed system; however one needs to consider the mentioned possibility of co-integration in a single chip with other MEMS inertial devices, which would dramatically reduce the area wasted for packaging and stacking.

Offsets in the order of few $\mathrm{mT}$ were tuned down to the $\mu T$ range, which is far better than offset values of several available devices. Achieved Allan stability in the order of 70-150 nT at few tens seconds were observed: this finding is hard to compare with available products, as in no data-sheet the offset stability is provided. Similar results were obtained for other kinds of Lorentz force MEMS devices as reported in [14], while FM magnetometers with current chopping achieved slightly better results [2].

The presented preliminary investigation of offset sources generates forthcoming research on techniques for offset suppression at its origin, with the final purpose of further improving the output stability.

\section{REFERENCES}

[1] G. Laghi, A. Longoni, P. Minotti, A. Tocchio, and G. Langfelder, "100 $\mu A, 320 \mathrm{nT} / \sqrt{\mathrm{Hz}}, 3$-axis Lorentz Force MEMS Magnetometer," in Solid-State Sensors, Actuators and Microsystems (TRANSDUCERS), 2015 Transducers - 2015 18th International Conference on, June 2015, pp. 803-806.

[2] M. Li, S. Sonmezoglu, and D. Horsley, "Extended Bandwidth Lorentz Force Magnetometer Based on Quadrature Frequency Modulation," Microelectromechanical Systems, Journal of, 2014.

[3] J. Kyynarainen, J. Saarilahti, H. Kattelus, A. Karkkainen, T. Meinander, A. Oja, P. Pekko, H. Seppa, M. Suhonen, H. Kuisma, S. Ruotsalainen, and M. Tilli, "A 3D micromechanical compass," Sensors and Actuators A: Physical, vol. 142, no. 2, pp. 561-568, 2008.

[4] H. Emmerich, M. Schofthaler, and U. Knauss, "A novel micromachined magnetic-field sensor," in Micro Electro Mechanical Systems, 1999. MEMS '99. Twelfth IEEE International Conference on, Jan 1999, pp. 94-99.

[5] W.-M. Lai, F.-M. Hsu, W.-L. Sung, R. Chen, and W. Fang, "Monolithic integration of micro magnetic pillar array with anisotropic magnetoresistive (AMR) structure for out-of-plane magnetic field detection," in Micro Electro Mechanical Systems (MEMS), 2015 28th IEEE International Conference on, Jan 2015, pp. 901-904.

[6] C. Sander, R. Raz, P. Ruther, O. Paul, T. Kaufmann, M. Cornils, and M. Vecchi, "Fully symmetric vertical hall devices in CMOS technology," in SENSORS, 2013 IEEE, Nov 2013, pp. 1-4.

[7] M. J. Thompson and D. A. Horsley, "Parametrically amplified z axis lorentz force magnetometer," Journal of Microelectromechanical Systems, vol. 20, no. 3, pp. 702-710, June 2011.

[8] E. Mehdizadeh, V. Kumar, and S. Pourkamali, "Sensitivity enhancement of lorentz force mems resonant magnetometers via internal thermalpiezoresistive amplification," IEEE Electron Device Letters, vol. 35, no. 2, pp. 268-270, Feb 2014.

[9] S. K. S. Choi, Y. Yoon and M. Allen, "Nonlinear sensitivity enhancement of resonant microsensors and its application to low powermagnetic sensing," Journal of Micromechanichs and Microengineering, vol. 21, p. 045004, June 2011.

[10] S. Zotov, B. Simon, A. Trusov, and A. Shkel, "High Quality Factor Resonant MEMS Accelerometer With Continuous Thermal Compensation," Sensors Journal, IEEE, vol. 15, no. 9, pp. 5045-5052, Sept 2015.

[11] Y. Zhao, J. Zhao, X. Wang, G. M. Xia, A. P. Qiu, Y. Su, and Y. P. $\mathrm{Xu}$, "A Sub- $\mu \mathrm{g}$ Bias-Instability MEMS Oscillating Accelerometer With an Ultra-Low-Noise Read-Out Circuit in CMOS," Solid-State Circuits, IEEE Journal of, vol. 50, no. 9, pp. 2113-2126, Sept 2015.

[12] G. Langfelder, S. Dellea, A. Berthelot, P. Rey, A. Tocchio, and A. Longoni, "Analysis of Mode-Split Operation in MEMS Based on Piezoresistive Nanogauges," Microelectromechanical Systems, Journal of, vol. 24, no. 1, pp. 174-181, Feb 2015.

[13] V. Kempe, Inertial MEMS: Principles and Practice. Cambridge University Press, 2011. 
TABLE 2. COMPARISON OF THE PRESENTED MAGNETIC FIELD SENSING SYSTEM PERFORMANCE WITH THE STATE-OF-THE-ART

\begin{tabular}{|c|c|c|c|c|c|c|c|}
\hline Magnetometer & Technology & $\begin{array}{c}\text { Demonstrated } \pm \text { FSR } \\
{[\mathrm{mT}]}\end{array}$ & $\begin{array}{l}\text { Current* } \\
{\left[\mu A_{r m s}\right]}\end{array}$ & $\begin{array}{c}\text { Demonstrated max BW } \\
{[\mathrm{Hz}]}\end{array}$ & $\begin{array}{c}\text { FOM }^{*} \\
{\left[\mu \mathrm{T} \cdot \mu A_{r m s} / \sqrt{\mathrm{Hz}}\right]}\end{array}$ & $\begin{array}{c}\text { Footprint } \\
{\left[\mathrm{mm}^{2}\right]}\end{array}$ & $\begin{array}{l}\text { Offset } \\
{[\mu \mathrm{T}]}\end{array}$ \\
\hline STM LIS3MDL [37] & $\mathrm{AMR}^{* *}$ & 1.2 & 90 & 10 & 11.4 & $2 \times 2$ & 100 \\
\hline Freescale MAG3110 [42] & MTJ $^{* *}$ & 1.0 & 300 & 40 & 19.0 & $2 \times 2$ & 100 \\
\hline (in low power mode) & & & 3 & 0.04 & 3.6 & & \\
\hline Asahi Kasei AK8975 [43] & Hall $^{* *}$ & 1.2 & 117 & 4 & 17.5 & $2 \times 2$ & 300 \\
\hline Honeywell HMC5883 [44] & $\mathrm{AMR}^{* *}$ & 0.8 & 33 & 3.75 & 3.4 & $3 \times 3$ & N/A \\
\hline Bosch BMM150 [45] & AMR+Hall** & 1.3 & 1630 & 10 & 155 & $1.56 \times 1.56$ & $2 \mathrm{SW}$ calibration \\
\hline (in low power mode) & & & 57 & 5 & 35.6 & & \\
\hline Emmerich [4] & MEMS & 5.0 & $1000^{* * *}$ & 6 & 1200 & N/A & N/A \\
\hline Kynnarainen [3] & MEMS** & 0.2 & $100^{* * *}$ & 2.5 & 7 & $>3.4 \times 3.4$ & 25 \\
\hline $\mathrm{Li}[2]$ & MEMS & 3.6 & $900^{* * *}$ & 50 & 640 & $0.4 \times 0.4^{+}$ & 22 \\
\hline Langfelder [18] & MEMS & 6.0 & $50^{* * *}$ & 160 & 170 & $1.1 \times 0.2^{+}$ & N/A \\
\hline $\operatorname{Li}[46]$ & MEMS & 8.0 & $4000^{* * *}$ & N/A & 80000 & $1.2 \times 0.7^{+}$ & N/A \\
\hline Minotti [23] & MEMS & 2.4 & 150 & 150 & 58 & $1.7 \times 0.8^{+}$ & N/A \\
\hline $\operatorname{Laghi}[1]^{x}$ & MEMS** & 3.0 & $33^{* * *}$ & 39 & 32 & $4 \times 4^{++}$ & 2 \\
\hline This work & MEMS** & 5.5 & $33^{* * *}$ & 55 & 6.6 & $4 \times 4^{++}$ & 2 \\
\hline
\end{tabular}

${ }^{*}$ Parameter normalized to a single axis (overall current is divided by 3). For 3-axis devices, the worst axis performance is reported

*** 3 -axis device.

*** ASIC consumption not included.

+ Footprint of the sensor only.

${ }^{++}$Footprint of the sensor and the full-package (ASIC is assumed to be stacked on top).

$\mathrm{x}[1]$ is preliminary characterization with respect to this work (no simultaneous operation of the three axes, different package pressure, different electronics).

[14] M. Li and D. Horsley, "Offset Suppression in a Micromachined Lorentz Force Magnetic Sensor by Current Chopping," Microelectromechanical Systems, Journal of, vol. 23, no. 6, pp. 1477-1484, Dec 2014.

[15] F. Giacci, S. Dellea, A. Longoni, and G. Langfelder, "Vibrations rejection in gyroscopes based on piezoresistive nanogauges," in SolidState Sensors, Actuators and Microsystems (TRANSDUCERS), 2015 Transducers - 2015 18th International Conference on, June 2015, pp. 780-783.

[16] W. Tang, T.-C. Nguyen, and R. Howe, "Laterally driven polysilicon resonant microstructures," in Micro Electro Mechanical Systems, 1989, Proceedings, An Investigation of Micro Structures, Sensors, Actuators, Machines and Robots. IEEE, Feb 1989, pp. 53-59.

[17] C.-C. Nguyen and R. Howe, "An integrated CMOS micromechanical resonator high-Q oscillator," Solid-State Circuits, IEEE Journal of, vol. 34, no. 4, pp. 440-455, Apr 1999.

[18] G. Langfelder, G. Laghi, P. Minotti, A. Tocchio, and A. Longoni, "Off-Resonance Low-Pressure Operation of Lorentz Force MEMS Magnetometers," Industrial Electronics, IEEE Transactions on, vol. 61, no. 12, pp. 7124-7130, Dec 2014.

[19] G. Langfelder and A. Tocchio, "Operation of Lorentz-Force MEMS Magnetometers With a Frequency Offset Between Driving Current and Mechanical Resonance," Magnetics, IEEE Transactions on, vol. 50, no. 1, pp. 1-6, Jan 2014.

[20] G. Langfelder, A. Caspani, and A. Tocchio, "Design Criteria of LowPower Oscillators for Consumer-Grade MEMS Resonant Sensors," Industrial Electronics, IEEE Transactions on, vol. 61, no. 1, pp. 567574, Jan 2014.

[21] G. Langfelder, S. Dellea, F. Zaraga, D. Cucchi, and M. Urquia, "The Dependence of Fatigue in Microelectromechanical Systems on the Environment and the Industrial Packaging," Industrial Electronics, IEEE Transactions on, vol. 59, no. 12, pp. 4938-4948, Dec 2012.

[22] H. Weinberg, "Gyro Mechanical Performance: The Most Important Parameter," Analog Devices Technical Article, vol. 2158, pp. 49384948, Sept 2011.

[23] P. Minotti, S. Brenna, G. Laghi, A. Bonfanti, G. Langfelder, and A. Lacaita, "A Sub-400-nT/ $\sqrt{H z}, 775-\mu \mathrm{W}$, Multi-Loop MEMS
Magnetometer With Integrated Readout Electronics," Microelectromechanical Systems, Journal of, vol. 24, no. 6, pp. 1938-1950, Dec 2015.

[24] M. Turner, "Verification of particle-in-cell simulations with monte carlo collisions against exact solutions of the boltzmann-poisson equations," in Plasma Sciences (ICOPS), 2015 IEEE International Conference on, May 2015, pp. 1-1.

[25] A. Frangi, G. Laghi, G. Langfelder, P. Minotti, and S. Zerbini, "Optimization of Sensing Stators in Capacitive MEMS Operating at Resonance," Microelectromechanical Systems, Journal of, vol. 24, no. 4, pp. 1077-1084, Aug 2015.

[26] B. Simon, A. Trusov, and A. Shkel, "Anti-phase mode isolation in tuning-fork MEMS using a lever coupling design," in Sensors, 2012 IEEE, Oct 2012, pp. 1-4.

[27] A. Sharma, M. Zaman, and F. Ayazi, "A 104-db dynamic range transimpedance-based cmos asic for tuning fork microgyroscopes," Solid-State Circuits, IEEE Journal of, vol. 42, no. 8, pp. 1790-1802, Aug 2007.

[28] J. Raman, E. Cretu, P. Rombouts, and L. Weyten, "A Closed-Loop Digitally Controlled MEMS Gyroscope With Unconstrained SigmaDelta Force-Feedback," Sensors Journal, IEEE, vol. 9, no. 3, pp. 297305, March 2009.

[29] P. Pouliquen, J. Vogelstein, and R. Etienne-Cummings, "Practical considerations for the use of a Howland current source for neurostimulation," in Biomedical Circuits and Systems Conference, 2008. BioCAS 2008. IEEE, Nov 2008, pp. 33-36.

[30] B. Kim, M. Hopcroft, R. Candler, C. Jha, M. Agarwal, R. Melamud, S. A. Chandorkar, G. Yama, and T. Kenny, "Temperature Dependence of Quality Factor in MEMS Resonators," Microelectromechanical Systems, Journal of, vol. 17, no. 3, pp. 755-766, June 2008.

[31] S. Sonmezoglu and D. A. Horsley, "Off-resonance operation of a mems lorentz force magnetometer with improved thermal stability of the scale factor," in 2016 IEEE 29th International Conference on Micro Electro Mechanical Systems (MEMS), Jan 2016, pp. 103-106.

[32] Invensense Inc., "MPU-9255, Product Specification," [online]. Available: http://www.invensense.com, accessed Nov. 2015, Nov 2015.

[33] S. Tumanski, Thin Film Magnetoresistive Sensors. Taylor \& Francis, 
2001.

[34] M. Thompson and D. Horsley, "Parametrically amplified MEMS magnetometer," in Solid-State Sensors, Actuators and Microsystems Conference, 2009. TRANSDUCERS 2009. International, June 2009, pp. 1194 1197.

[35] M. Li, V. T. Rouf, G. Jaramillo, and D. A. Horsley, "Mems lorentz force magnetic sensor based on a balanced torsional resonator," in Solid-State Sensors, Actuators and Microsystems (TRANSDUCERS EUROSENSORS XXVII), 2013 Transducers Eurosensors XXVII: The 17th International Conference on, June 2013, pp. 66-69.

[36] G. Langfelder, A. Tocchio, and D. Paci, "Magnetic sensor including a lorentz force transducer driven at a frequency different from the resonance frequency, and method for driving a lorentz force transducer," Patent TO2013A000 653, US2015/0 035 526A1, 07 31, 2013.

[37] ITmems s.r.1., "MCP-G, Product Specification," [online]. Available: http://www.itmems.it, accessed Nov. 2015, Nov 2015.

[38] J. Wu, G. Fedder, and L. Carley, "A low-noise low-offset capacitive sensing amplifier for a 50- mu;g/ radic; hz monolithic cmos mems accelerometer," Solid-State Circuits, IEEE Journal of, vol. 39, no. 5, pp. 722-730, May 2004.

[39] R. Antonello, R. Oboe, L. Prandi, C. Caminada, and F. Biganzoli, "Open loop compensation of the quadrature error in mems vibrating gyroscopes," in Industrial Electronics, 2009. IECON '09. 35th Annual Conference of IEEE, Nov 2009, pp. 4034-4039.

[40] E. Tatar, S. Alper, and T. Akin, "Quadrature-error compensation and corresponding effects on the performance of fully decoupled mems gyroscopes," Microelectromechanical Systems, Journal of, vol. 21, no. 3, pp. 656-667, June 2012.

[41] M. Li, V. Rouf, M. Thompson, and D. Horsley, "Three-axis lorentzforce magnetic sensor for electronic compass applications," Microelectromechanical Systems, Journal of, vol. 21, no. 4, pp. 1002-1010, Aug 2012.

[42] Freescale, "Mag3110, Product Specification," [online]. Available: http://www.nxp.com, accessed Nov. 2015, Nov 2015.

[43] Asahi Kasei, “AK8975, Product Specification," [online]. Available: http://www.asahi-kasei.co.jp/asahi/en, accessed Nov. 2015, Nov 2015.

[44] Honeywell, "HMC5883, Product Specification," [online]. Available: http://www.honeywell.com, accessed Nov. 2015, Nov 2015.

[45] Robert Bosch Gmbh, "BMM150, Product Specification," [online]. Available: https://www.bosch-sensortec.com, accessed Nov. 2015, Nov 2015.

[46] M. Li, S. Nitzan, and D. Horsley, "Frequency-Modulated Lorentz Force Magnetometer With Enhanced Sensitivity via Mechanical Amplification," Electron Device Letters, IEEE, vol. 36, no. 1, pp. 62-64, Jan 2015.

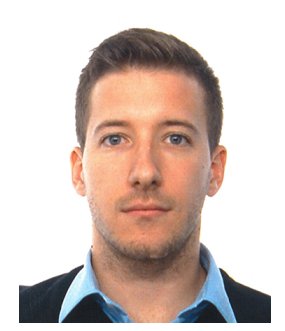

Giacomo Laghi received the B.Sc. and M.Sc. degrees in Electronics Engineering from Politecnico di Milano, Milano, Italy, in 2011 and 2013, respectively.

Since November 2013 he has been a Ph.D. student and teaching assistant with the Laboratory of MEMS and Microsensors, DEIB, Politecnico di Milano, being involved in European projects and industrial partnerships. His research interests include the design of MEMS inertial sensors and related electronics, with a particular focus on Lorentz-Force magnetometers and the study of damping phenomena in MEMS packages.

$\mathrm{He}$ is co-author of about 10 publications in the field.

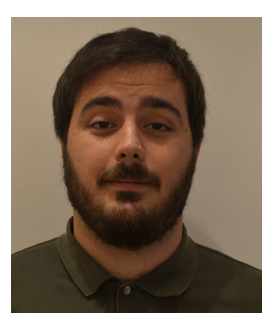

Cristiano R. Marra was born in Larino (CB), Italy, in 1990. He received both the B.Sc. and the M.Sc. degrees in Electronics Engineering from Politecnico di Milano, in 2012 and 2015 respectively.

Since November 2015, he has been a Ph.D. Student in Information Technologies with the Laboratory of MEMS and Microsensors, Politecnico di Milano. His area of interests includes microelectromechanical sensors and systems, in particular magnetic field detectors and accelerometers, and related electronics.

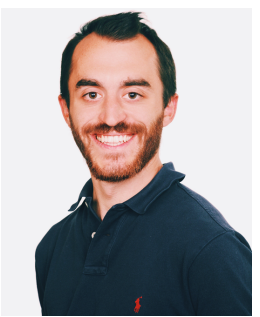

Paolo Minotti received the B.S. and M.S. degrees in electronics engineering from Politecnico di Milano, Italy, in 2010 and 2013, respectively.

$\mathrm{He}$ is currently pursuing the Ph.D. degree with the Department of Electronics, Information Technology, and Bioengineering, Politecnico di Milano. His research interests include microelectromechanical systems, in particular, gyroscopes and magnetic sensors, with a focus on ASIC design. He is the author of about 10 publications in the field.

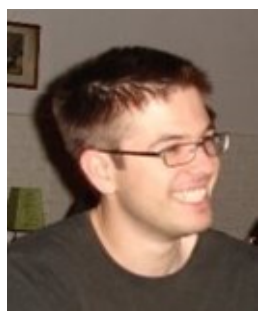

Alessandro Tocchio received the Ph.D. degree in information technology from the Politecnico di Milano, Italy, in 2012.

His research interests include the design and fabrication of novel microelectromechanical sensors, and the development of the related read out electronics with a special focus on low-power low-noise circuit topologies.

Dr. Tocchio moved to ST Microelectronics, Italy, in 2012, where he is currently a Senior MEMS Designer. He has authored about 30-refereed scientific publications and several patents in the field of microsystems.

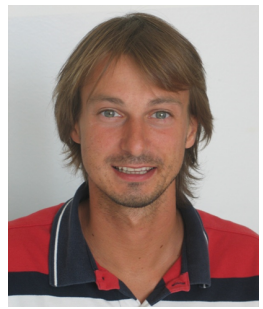

Giacomo Langfelder received the Ph.D. degree in Information Technology from Politecnico di Milano, Italy, in 2009, where he is currently an Assistant Professor of Digital Imaging and of MEMS, and Microsensors.

His recent research develops on CMOS sensors, MEMS inertial sensors, and related characterization and operation electronics, with a marked focus on reliability aspects. He has authored about 90 refereed international publications and is the Co-Inventor of six patents in the field of sensors.

Dr. Langfelder was a recipient of the Premio di Laurea Accenture in 2005 and the Premio per la Promozione della Ricerca Scientifica in 2011, granted by Rotary International. 